\title{
ON THE THEORY OF EXPONENTIAL GROUPS $\left({ }^{1}\right)$
}

\author{
BY \\ LAJOS PUKANSZKY
}

1. Introduction. Let $\mathscr{L}$ be a real solvable Lie algebra. As it is known, there exists a sequence of ideals $\mathscr{L}_{0}=\mathscr{L} \supset \mathscr{L}_{1} \supset \ldots \supset \mathscr{L}_{n}=(0)$ of $\mathscr{L}$, such that the codimension of $\mathscr{L}_{i+1}$ in $\mathscr{L}_{i}$ is 1 or $2(i=0,1,2, \ldots, m-1)$, and such that in the latter case the representation induced by the adjoint representation of $\mathscr{L}$ on $\mathscr{L}_{i} / \mathscr{L}_{i+1}$ is irreducible. If for some $i=0,1,2, \ldots, m-1$, the codimension of $\mathscr{L}_{i+1}$ in $\mathscr{L}_{i}$ is 1 , and if $l_{0}$ is a nonzero element of $\mathscr{L}_{i}$ not contained in $\mathscr{L}_{i+1}$, we have for all $l$ in $\mathscr{L}$

$$
\operatorname{ad} l\left(l_{0}\right)=\gamma(l) l_{0} \quad\left(\bmod \mathscr{L}_{i+1}\right)
$$

where $\gamma(l)$ is a linear form vanishing on the first derived algebra of $\mathscr{L}$. If the codimension of $\mathscr{L}_{i+1}$ in $\mathscr{L}_{i}$ is 2 , there exists a pair of elements $l^{\prime}, l^{\prime \prime}$, linearly independent $\bmod \mathscr{L}_{i+1}$, of $\mathscr{L}_{i}$ such that for all $l$ in $\mathscr{L}$

$$
\begin{aligned}
& \text { ad } l\left(l^{\prime}\right)=\gamma^{\prime}(l) l^{\prime}-\gamma^{\prime \prime}(l) l^{\prime \prime} \\
& \text { ad } l\left(l^{\prime \prime}\right)=\gamma^{\prime \prime}(l) l^{\prime}+\gamma^{\prime}(l) l^{\prime \prime}
\end{aligned} \quad\left(\bmod \mathscr{L}_{i+1}\right),
$$

where $\gamma^{\prime}$ and $\gamma^{\prime \prime}$ are linear forms on $\mathscr{L}$ of the kind considered above. The collection of all linear forms $\left\{\gamma(l), \gamma^{\prime}(l) \pm i \gamma^{\prime \prime}(l)\right\}$ where $\gamma, \gamma^{\prime}, \gamma^{\prime \prime}$ respectively have been obtained in the indicated fashion, is called the system of roots of $\mathscr{L}$. The set of values corresponding to a fixed $l$ in $\mathscr{L}$ coincides with the collection of eigenvalues of the operator ad $l$ on $\mathscr{L}$.

We say that a real solvable Lie algebra is exponential if its complex roots are of the form $(1+i \alpha) \delta(l)$, where $\alpha$ is a nonzero real number and $\delta(l)$ is a real linear form on $\mathscr{L}$. We shall call a connected and simply connected Lie group exponential, if its Lie algebra is exponential. Let $\mathscr{L}$ be a solvable Lie algebra and $G$ the corresponding connected and simply connected Lie group.

It is known (cf. [2] and [8]) that the exponential map establishes an analytic isomorphism between the underlying manifolds of $\mathscr{L}$ and $G$ resp. if and only if $\mathscr{L}$ is exponential. One sees easily, that any subalgebra or factoralgebra of an exponential Lie algebra is again of the same kind.

An important special case is that of a nilpotent Lie algebra, which is characterized by the vanishing of all roots.

The study of the classification of all irreducible unitary representations of an exponential group was started by J. Dixmier's work on nilpotent groups, continued

Received by the editors July $11,1966$.

( $\left.{ }^{1}\right)$ Prepared with partial support from Nonr-551 (57). 
later by A. A. Kirillov (for a systematic account cf. [7]). The first result referring to not necessarily nilpotent exponential groups is due to O. Takenouchi, who showed that the factor representations of exponential groups are of type I (cf. [9]). In a recent work [1] P. Bernat gives a detailed study of the system of all irreducible unitary representations of an exponential group. His results, extending those found in the nilpotent case by Kirillov, can be summed up as follows. Let $\mathscr{L}$ be an exponential Lie algebra and $\mathscr{L}^{\prime}$ the dual of the underlying space of $\mathscr{L}$; we denote by $\left(l, l^{\prime}\right)\left(l \in \mathscr{L}, l^{\prime} \in \mathscr{L}^{\prime}\right)$ the canonical bilinear form on $\mathscr{L} \times \mathscr{L}^{\prime}$. Let $l_{0}^{\prime}$ be a fixed element of $\mathscr{L}^{\prime}$ and $H$ a subalgebra of $\mathscr{L}$. We say, that $H$ is subordinated to $l_{0}^{\prime}$, if the first derived algebra of $H$ is orthogonal to $l_{0}^{\prime}$; in this case we shall write $H<l_{0}^{\prime}$. Let us denote by $\overline{\mathscr{L}}$ the connected and simply connected Lie group belonging to $\mathscr{L}$, and by $\bar{H}$ the connected subgroup corresponding to the subalgebra $H \subset \mathscr{L}$. If $H<l_{0}^{\prime}$, the formula

$$
\chi(\exp h)=\exp \left(i\left(h, l_{0}^{\prime}\right)\right) \quad(h \in H)
$$

defines a character of $\bar{H}$. Let us denote by ind $\left(H, l_{0}^{\prime}\right)$ the unitary representation of $\overline{\mathscr{L}}$ induced by the character $\chi$ of $\bar{H}$. Takenouchi showed, using G. W. Mackey's results on systems of imprimitivity, that any irreducible unitary representation of $\overline{\mathscr{L}}$ can be written in the form ind $\left(H, l_{0}^{\prime}\right)$, by choosing $H$ and $l_{0}^{\prime}$ appropriately. Bernat proves first that, conversely, for any given $l_{0}^{\prime}$ in $\mathscr{L}^{\prime}$ there exists at least one choice of the subalgebra $H$, subordinated to $l_{0}^{\prime}$, for which ind $\left(H, l_{0}^{\prime}\right)$ turns out to be irreducible. We denote by $\sigma$ the adjoint representation of $\overline{\mathscr{L}}$ acting on $\mathscr{L}$, and by $\delta$ the representation, which is contragredient to $\sigma$, of $\overline{\mathscr{L}}$ on $\mathscr{L}^{\prime}$; that is, for any $g \in \overline{\mathscr{L}}$ the operators $\delta(g)$ and $\sigma(g)$ are connected by the relation

$$
\left(\sigma\left(g^{-1}\right) l, l^{\prime}\right)=\left(l, \delta(g) l^{\prime}\right) \quad\left(l \in \mathscr{L}, l^{\prime} \in \mathscr{L}^{\prime}\right) .
$$

Suppose now that $H_{0}<l_{0}^{\prime}$ and $H_{1}<l_{1}^{\prime}$ such that ind $\left(H_{0}, l_{0}^{\prime}\right)$ and ind $\left(H_{1}, l_{1}^{\prime}\right)$ are irreducible. Then we have ind $\left(H_{0}, l_{0}^{\prime}\right)=$ ind $\left(H_{1}, l_{1}^{\prime}\right)$, the equality sign indicating the unitary equivalence of the corresponding representations, if and only if there exists an element $g$ of $\overline{\mathscr{L}}$ such that $l_{1}^{\prime}=\delta(g) l_{0}^{\prime}$; in other words, if and only if $l_{0}^{\prime}$ and $l_{1}^{\prime}$ belong to the same orbit of $\mathscr{L}^{\prime}$ with respect to $\delta$. In order that, for a given $l_{0}^{\prime}$, the representation ind $\left(H, l_{0}^{\prime}\right)$ be irreducible, it is necessary that $H$ should be of a maximal dimension in the family of subalgebras subordinated to $l_{0}^{\prime}$. Furthermore, for such an $H$, dim $H$ depends only on the orbit determined by $l_{0}^{\prime}$. Denoting this by $o\left(l_{0}^{\prime}\right)$, Kirillov proved that in the nilpotent case the dimension in question is given by $\operatorname{dim} \mathscr{L}-\frac{1}{2} \operatorname{dim} o\left(l_{0}^{\prime}\right)$ [3, Lemma 5.2]. In the general case, however, the maximality of the dimension of $H$ is far from being sufficient to imply the irreducibility of ind $\left(H, l_{0}^{\prime}\right)$; in fact, already the consideration of the affine group of the real line furnishes counterexamples. As Bernat shows, a sufficient condition assuring that the maximality of $\operatorname{dim} H$ implies the irreducibility of ind $\left(H, l_{0}^{\prime}\right)$ is that all real roots of $\mathscr{L}$ be zero. An exponential Lie algebra satisfying this condition is called quasinilpotent; evidently a nilpotent algebra is quasi-nilpotent. 
The purpose of the present paper is to give necessary and sufficient conditions on the subalgebra $H$, in order that ind $\left(H, l_{0}^{\prime}\right)$ turn out to be irreducible. To describe these we denote by $H^{\perp}$ the orthogonal complement of $H$ in $\mathscr{L}^{\prime}$. Then we have first the following result (cf. Theorem 1): ind $\left(H, l_{0}^{\prime}\right)$ is irreducible if and only if $H$ is of a maximal dimension among the subalgebras subordinated to $l_{0}^{\prime}$, and the linear variety $l_{0}^{\prime}+H^{\perp}$ is contained in $o\left(l_{0}^{\prime}\right)$ (=the orbit of $l_{0}^{\prime}$ ). We show (cf. Lemma 5), that the first of these conditions, similarly as in the case of nilpotent groups, is equivalent to $\operatorname{dim} H=\operatorname{dim} \mathscr{L}-\frac{1}{2} \operatorname{dim} o\left(l_{0}^{\prime}\right)$. As far as the necessity of the second condition is concerned, this follows immediately from the results of Bernat quoted above. In fact, to this end it suffices to observe that evidently

$$
\text { ind }\left(H, l_{0}^{\prime}\right)=\text { ind }\left(H, l_{0}+h^{\perp}\right)
$$

for any choice of $h^{\perp}$ in $H^{\perp}$, and therefore, if these representations are irreducible, then $l_{0}^{\prime}$ and $l_{0}^{\prime}+h^{\perp}$ must lie on the same orbit. Next we prove (cf. Proposition 3) that corresponding to Bernat's observation the first condition implies the second one if $\mathscr{L}$ is quasi-nilpotent. Another situation, where the same implication is true, is furnished by an $l_{0}^{\prime}$, for which the orbit $o\left(l_{0}^{\prime}\right)$ is closed (cf. Proposition 4). Since for a nilpotent $\mathscr{L}$ all orbits of $\mathscr{L}^{\prime}$ according to $\delta$ are closed (even Zariski closed; cf. e.g., [7, Deuxième Partie, Chapitre I, §2]), the sufficiency to yield irreducibility, of $\operatorname{dim} H=\operatorname{dim} \mathscr{L}-\frac{1}{2} \operatorname{dim} o\left(l_{0}^{\prime}\right)$ obtains here a new proof. We repeat, however, that in the general case the two conditions are independent of each other (cf. Remark 2 after Definition 2 in §3).

Theorem 2 shows that in the case of solvable algebras with real roots the necessary and sufficient condition of Theorem 1 can be alternatively described in close analogy with finite groups. For this we recall first the following theorem of Shoda (generalized by Mackey, cf. [5, Theorem 6]). Let $G$ be a finite group, $\Gamma$ a subgroup of $G$, and $\chi$ a homomorphism of $\Gamma$ into the multiplicative group of complex numbers of absolute value one. For an element $x$ of $G$, we denote by $\Gamma_{x}$ the intersection of the subgroups $\Gamma$ and $x^{-1} \Gamma x$, and by $\chi_{x}$ the character of $\Gamma_{x}$ defined by $\chi_{x}(\gamma)=\chi\left(x \gamma x^{-1}\right)\left(\gamma \in \Gamma_{x}\right)$. Then the representation, induced by $\chi$, of $G$ turns out to be irreducible if and only if for all $x$ not belonging to $\Gamma, \chi_{x}$ is different from the restriction of $\chi$ to $\Gamma_{x}$. Let $G$ be the connected and simply connected Lie group which belongs to the solvable Lie algebra $\mathscr{L}$ with real roots. Suppose again that $H$ is a subalgebra, subordinated to $l_{0}^{\prime}$, and denote by $\bar{H}$ the connected subgroup determined by $H$. Theorem 2 asserts that ind $\left(H, l_{0}^{\prime}\right)$ is an irreducible unitary representation of $G$ if and only if the condition to yield irreducibility of the above theorem is fulfilled upon replacing $\Gamma$ by $\bar{H}$ and $\chi$ by the character

$$
\chi(\exp h)=\exp \left[i\left(h, l_{0}^{\prime}\right)\right] \quad(h \in H)
$$

of $\bar{H}$ resp.

The reader is assumed to be familiar with the basic properties of induced representations, as exposed in [6]; we shall also use several reasonings of Takenouchi 
[9], to be specified later. On the other hand, it is necessary for our purposes to reproduce the relevant parts of Bernat's paper [1] with some variations, and therefore, on this side, we shall use but a small number of elementary computations without proof. For the same reason, we give a complete proof of Theorem 1.

The author gratefully acknowledges the inspiration he received through the lectures of B. Kostant at Haverford College in December of 1965.

2. Notations. Besides those explained in the Introduction, we shall make constant use of the following notations.

a. Let $V$ be a finite dimensional vector space over the real field and $B\left(v_{1}, v_{2}\right)$ $\left(v_{1}, v_{2} \in V\right)$ a skew symmetric bilinear form on $V$. For a subspace $W$ of $V$ we denote by $W_{B}^{\perp}$ the orthogonal complement of $W$ in $V$ with respect to $B$. The subspace $R=V_{B}^{\perp}$ is called the radical of $B$. We shall often use the following elementary relation, valid for any choice of the subspace $W$ of $V$ :

$$
\operatorname{dim} W_{B}^{\perp}=\operatorname{dim} V-\operatorname{dim} W+\operatorname{dim}(W \cap R) .
$$

$W$ is called self orthogonal if $W \subseteq W_{B}^{\perp}$, and maximal self orthogonal if $W=W_{B}^{\perp}$. One verifies easily by aid of the above formula that a self orthogonal subspace $W$ is maximal if and only if

$$
\operatorname{dim} W=\frac{1}{2}(\operatorname{dim} V+\operatorname{dim} R) .
$$

b. All skew symmetric bilinear forms considered in this paper are going to be obtained by taking a Lie algebra $\mathscr{L}$, by fixing an element $l_{0}^{\prime}$ of the dual $\mathscr{L}^{\prime}$ of the underlying space of $\mathscr{L}$, and by putting

$$
B\left(l_{1}, l_{2}\right)=\left(\left[l_{1}, l_{2}\right], l_{0}^{\prime}\right) \quad\left(l_{1}, l_{2} \in \mathscr{L}\right) .
$$

The expressions self orthogonal and maximal self orthogonal with respect to $l_{0}^{\prime}$ have to be interpreted through the bilinear form determined by $l_{0}^{\prime}$. Its radical will be denoted by $R\left(l_{0}^{\prime}\right)$, or simply by $R$, if its dependence on $l_{0}^{\prime}$ is indicated by the context. Let $\mathscr{L}_{1}$ be a subspace of $\mathscr{L}$; then $B\left(l_{1}, l_{2} ; \mathscr{L}_{1}, l_{0}^{\prime}\right)$ will stand for the restriction of the above bilinear form to $\mathscr{L}_{1}$ (if $\mathscr{L}_{1}=\mathscr{L}$, it will not be indicated).

c. Let $H$ be a subspace of $\mathscr{L}$; then, as explained in a, $H_{B}^{\perp}$ will denote the orthogonal complement of $H$ in $\mathscr{L}$ with respect to the bilinear form $B$. On the other hand, $H^{\perp}$ indicates the orthogonal complement of $H$ in the dual of $\mathscr{L}$ with respect to the canonical bilinear form on $\mathscr{L} \times \mathscr{L}^{\prime}$.

d. All Lie algebras considered in this paper are assumed to be exponential. If $\mathscr{L}$ is a Lie algebra, we denote by $\overline{\mathscr{L}}$ the corresponding connected and simply connected Lie group. Similarly, for any subalgebra $H$ of $\mathscr{L}, \bar{H}$ will stand for the connected subgroup determined by $H$. Finally, if $l$ is some element of $\mathscr{L}$, we shall write $l$ for the element $\exp l$ of $\overline{\mathscr{L}}$.

3. Let $\mathscr{L}$ be a Lie algebra and $l_{0}^{\prime}$ a nonzero element of the dual of $\mathscr{L}$; all these will be kept fixed throughout this section. We shall write mostly $R$ for $R\left(l_{0}^{\prime}\right)$. 
One sees at once that $R$ is the collection of all elements of $\mathscr{L}$ satisfying $(\operatorname{ad} l)^{\prime} l_{0}^{\prime}=0$. Hence $R$ is a subalgebra of $\mathscr{L}$ and $g \in \bar{R}$ implies $\delta(g) l_{0}^{\prime}=l_{0}^{\prime}$. One can easily show, by making use of the assumption, according to which $\mathscr{L}$ is exponential, that conversely, if $\delta(g) l_{0}^{\prime}=l_{0}^{\prime}$ for some element $g$ of $\overline{\mathscr{L}}$, then $g$ belongs to $\bar{R}$, and thus the closed subgroup $\bar{R}$ of $\overline{\mathscr{L}}$ coincides with the stabilizer of $l_{0}^{\prime}$, but we are not going to use this fact directly. Sometimes $R$ too will be referred to as the stabilizer of $l_{0}^{\prime}$. Let us denote by $O$ the orbit $o\left(l_{0}^{\prime}\right)$ of $l_{0}^{\prime}$ in $\mathscr{L}^{\prime}$ according to the representation $\delta$ of $\mathscr{L}$. We have $\operatorname{dim} O=\operatorname{dim} \mathscr{L}-\operatorname{dim} R$; taking into account that the right hand side is just the rank of the skew symmetric bilinear form $B\left(l_{1}, l_{2} ; l_{0}^{\prime}\right)$, we can conclude that $\operatorname{dim} O$ is always even.

Suppose now that $H$ is a subalgebra of $\mathscr{L}$ satisfying $H<l_{0}^{\prime}$; that is, that $H$ is subordinated to $l_{0}^{\prime}$. Let us observe, incidentally, that this is equivalent to saying that $H$ is self orthogonal with respect to $l_{0}^{\prime}$ (cf. 2. b). If $h_{0}$ is some element of $H$, $\delta\left(\bar{h}_{0}\right) l_{0}^{\prime}-l_{0}^{\prime}$ is orthogonal to $H$. In fact, this is equivalent to $\left(h, \delta\left(\bar{h}_{0}\right) l_{0}^{\prime}\right)=\left(h, l_{0}^{\prime}\right)$ for all $h$ in $H$. The left hand side is the same as

$$
\left(\exp \left(-\left(\operatorname{ad} h_{0}\right)\right) h, l_{0}^{\prime}\right)=\left(h, l_{0}^{\prime}\right)+\sum_{j=1}^{\infty} \frac{(-1)^{j}}{j !}\left(\left(\operatorname{ad} h_{0}\right)^{j}(h), l_{0}^{\prime}\right)
$$

and to obtain the desired conclusion, it suffices to observe that $\left(\left(\operatorname{ad} h_{0}\right)^{j}(h), l_{0}^{\prime}\right)=0$ for $j>0$ if $H<l_{0}^{\prime}$.

Definition 1. Let $H$ be a subalgebra with $H<l_{0}^{\prime}$. We shall say that $H$ has the property $F$, and write $H=(F)$ if and only if

$$
l_{0}^{\prime}+H^{\perp}=\left\{\delta(\bar{h}) l_{0}^{\prime} ; h \in H\right\} .
$$

REMARK 1. We have just proved that the right hand side is always contained in the left hand side. The converse, however, is false even if the dimensions of the two sides coincide. In fact, let $\mathscr{L}$ be the Lie algebra with the basis $\left\{e_{0}, e_{1}\right\}$ satisfying $\left[e_{0}, e_{1}\right]=e_{1} ; \mathscr{L}$ is evidently exponential. If $l_{0}^{\prime}$ in $\mathscr{L}^{\prime}$ is such that $\left(e_{1}, l_{0}^{\prime}\right) \neq 0$, then $R=0$, and we have $H<l_{0}^{\prime}$ for any 1-dimensional subalgebra $H$. On the other hand $H=(F)$ if and only if $H=\left\{e_{1}\right\}$.

Before proceeding we observe (cf. 2a) that $H<l_{0}^{\prime}$ implies $H \leqq H_{B}^{\perp}$, hence (cf. 2a)

$\operatorname{dim} H \leqq \frac{1}{2}(\operatorname{dim} \mathscr{L}+\operatorname{dim}(H \cap R)) \leqq \frac{1}{2}(\operatorname{dim} \mathscr{L}+\operatorname{dim} R)=\operatorname{dim} \mathscr{L}-\frac{1}{2} \operatorname{dim} O$.

Definition 2. Suppose that $H$ is a subalgebra with $H<l_{0}^{\prime}$. We say that $H$ has the property $J$, and write $H=(J)$, if $1, \operatorname{dim} H=\operatorname{dim} \mathscr{L}-\frac{1}{2} \operatorname{dim} O, 2, l_{0}^{\prime}+H^{\perp} \subset O$ are satisfied at the same time.

REMARK 2. It is useful to observe that in general none of those two conditions implies the other. To see that 1 does not imply 2 , let $\mathscr{L}$ and $l_{0}^{\prime}$ be as in Remark 1 , and denote by $\left\{x_{0}, x_{1}\right\}$ the coordinates of an element $x$ of $\mathscr{L}^{\prime}$ with respect to the basis dual to $\left\{e_{0}, e_{1}\right\}$. Then

and thus

$$
O=o\left(l_{0}^{\prime}\right)=\left\{x ; \operatorname{sg}\left(x_{0}\right)=\operatorname{sg}\left(\left(e_{0}, l_{0}^{\prime}\right)\right)\right\}
$$

$$
\operatorname{dim} \mathscr{L}-\frac{1}{2} \operatorname{dim} O=1 \text {. }
$$


But for a 1-dimensional subalgebra $H$ the linear variety $l_{0}^{\prime}+H^{\perp}$ is contained in $O$ again if and only if $H=\left\{e_{1}\right\}$. To show that 2 does not imply 1 consider the Lie algebra with the basis $\left\{e_{1}, e_{2}, e_{3}\right\}$, satisfying $\left[e_{1}, e_{2}\right]=e_{3},\left[e_{1}, e_{3}\right]=\left[e_{2}, e_{3}\right]=0$; it is nilpotent, hence, in particular, exponential. Choose $l_{0}^{\prime}$ such that $\left(e_{3}, l_{0}^{\prime}\right) \neq 0$; then with notations as above $O=\left\{x ; x_{3}=\left(e_{3}, l_{0}^{\prime}\right)\right\}$, and thus $\operatorname{dim} \mathscr{L}-\frac{1}{2} \operatorname{dim} O=2$. On the other hand, defining $H$ by $H=\left\{e_{3}\right\}$, we have $l_{0}^{\prime}+H^{\perp}=O$, but $\operatorname{dim} H<2$. This example is a special case of the following situation. Suppose that $H$ is an ideal of $\mathscr{L}$ satisfying $R \subset H<l_{0}^{\prime}$. One can show that in this case the intersection of $O$ with $l_{0}^{\prime}+H^{\perp}$ is open in the latter; hence, if $O$ is closed, we have $l_{0}^{\prime}+H^{\perp} \subset O$.

Definition 3. Suppose that $H$ is a subalgebra with $H<l_{0}^{\prime}$. We say that $H$ has the property $G$, and write $H=(G)$, if $\operatorname{dim} H=\frac{1}{2}\left(\operatorname{dim} \mathscr{L}+\operatorname{dim} R\left(l_{0}^{\prime}+h^{\perp}\right)\right)$ for all elements $h^{\perp}$ in $H^{\perp}$.

Notation. Suppose that $\mathscr{L}_{0}$ is a subalgebra of $\mathscr{L}$, such that $H \subseteq \mathscr{L}_{0} \subseteq \mathscr{L}$. We shall write $H=\left(F, \mathscr{L}_{0}\right)$ to indicate that $H$ has the property $F$ as a subalgebra of $\mathscr{L}_{0}$ with respect to the element of $\mathscr{L}_{0}^{\prime}$, which is the restriction of $l_{0}^{\prime}$ to $\mathscr{L}_{0}$; similarly for properties $J$ and $G$.

Next we prove

Proposition 1. For any subalgebra $H$ of $\mathscr{L}$, the properties described in Definitions 1-3 are equivalent.

Proof. We shall carry it out in three steps.

A. $(F) \Rightarrow(J)$.

Since $l_{0}^{\prime}+H^{\perp} \subset O$ is trivially fulfilled, all that we have to show is that

$$
\operatorname{dim} H=\frac{1}{2}(\operatorname{dim} \mathscr{L}+\operatorname{dim} R),
$$

or what amounts to the same (cf. $2 \mathrm{a}, 2 \mathrm{~b}$ ), that $H$ is maximal self orthogonal with respect to $l_{0}^{\prime}$. We denote by $\rho$ the analytic map, defined for $h$ in $H$ by $\rho(h)=\delta(\bar{h}) l_{0}^{\prime}$, of the underlying space of $H$ into $l_{0}^{\prime}+H^{\perp}$. One sees easily that the rank of this map at the point $h_{0}$ of $H$ is the same as the dimension of the subspace $T\left(h_{0}\right)$ of $\mathscr{L}^{\prime}$ given by $T\left(h_{0}\right)=\left\{l^{\prime} ; l^{\prime}=\delta\left(\bar{h}_{0}\right)(\operatorname{ad} h)^{\prime} l_{0}^{\prime} ; h \in H\right\}$. Since $\operatorname{dim} T\left(h_{0}\right)=\operatorname{dim} T(0)$, this rank is constant. On the other hand, this must be the same as

$$
\operatorname{dim} H^{\perp}=\operatorname{dim} \mathscr{L}-\operatorname{dim} H,
$$

since, $H$ having the property $F$, the map $\rho$ is onto. Hence

$$
\operatorname{dim} T(0)=\operatorname{dim} H-\operatorname{dim}(H \cap R)=\operatorname{dim} \mathscr{L}-\operatorname{dim} H,
$$

and thus

$$
\operatorname{dim} H=\operatorname{dim} \mathscr{L}-\operatorname{dim} H+\operatorname{dim}(H \cap R)=\operatorname{dim} H_{B}^{\perp},
$$

$B$ indicating the skew symmetric bilinear form arising from $l_{0}^{\prime}$ (cf. 2b). Since $H<l_{0}^{\prime}$ we also have $H \subseteq H_{B}^{\perp}$, and thus finally $H=H_{B}^{\perp}$ which is the desired result.

B. $(J) \Rightarrow(G)$. 
If $H=(J)$, we have $l_{0}^{\prime}+H^{\perp} \subset O$, and the stabilizer of any element of the form $l_{0}^{\prime}+h^{\perp}$, with $h^{\perp}$ in $H^{\perp}$, has the same dimension. Thus,

$$
\frac{1}{2}(\operatorname{dim} \mathscr{L}+\operatorname{dim} R)=\operatorname{dim} H=\frac{1}{2}\left(\operatorname{dim} \mathscr{L}+\operatorname{dim} R\left(l_{0}^{\prime}+h^{\perp}\right)\right)
$$

for all $h^{\perp} \in H^{\perp}$; in other words, $H$ has the property $G$.

C. $(G) \Rightarrow(F)$.

The following reasonings will be organized with a view of other applications in the next sections, and, for the same reason, we shall prove slightly more than actually needed.

Lemma 1. Let $\mathscr{L}_{0}$ be a subalgebra, of codimension one, of $\mathscr{L}$ containing $H$, and suppose $H=\left(F, \mathscr{L}_{0}\right)$. Then we have $H=(F)$ if and only if $R\left(l_{0}^{\prime}+l_{0}^{\perp}\right)$ is contained in $\mathscr{L}_{0}$ for all $l_{0}^{\perp}$ in $\mathscr{L}_{0}^{\perp}$. The latter condition is automatically satisfied if $\mathscr{L}_{0}$ is an ideal and $R\left(l_{0}^{\prime}\right) \subseteq \mathscr{L}_{0}$.

Proof. I. To establish the necessity of the above condition first we observe that by virtue of A and $\mathrm{B}, H=(F)$ implies $H=(G)$. In this fashion $H$ is, in particular, maximal self orthogonal with respect to any element $l_{0}^{\prime}+l_{0}^{\perp}$, where $l_{0}^{\perp}$ is arbitrary in $\mathscr{L}_{0}^{\perp}$, since $H \subseteq \mathscr{L}_{0}$ implies $\mathscr{L}_{0}^{\perp} \subseteq H^{\perp}$. On the other hand, it is evident that $H_{B}^{\perp}$ contains the radical of B. Thus, finally $R\left(l_{0}^{\prime}+l_{0}^{\perp}\right) \subseteq H \subseteq \mathscr{L}_{0}$ for all $l_{0}^{\perp} \in \mathscr{L}_{0}$.

II. We shall prove the sufficiency in several steps. Observe that in what follows, to simplify the language, we shall often speak of $l_{0}^{\prime}$ instead of the restriction of $l_{0}^{\prime}$ to $\mathscr{L}_{0}$.

1. For $l_{0}^{1}=0$ we obtain $R=R\left(l_{0}^{\prime}\right) \subseteq \mathscr{L}_{0}$. Denote by $R_{0}$ the radical of $l_{0}^{\prime}$ in $\mathscr{L}_{0}$; evidently $R \cong R_{0}$. Since in any case $\operatorname{dim} \mathscr{L}-\operatorname{dim} R=\operatorname{dim} \mathscr{L}_{0}-\operatorname{dim} R_{0}+\eta(\eta=0,2)$, or $\operatorname{dim} R-\operatorname{dim} R_{0}=1$ or -1 , we have here $\operatorname{dim} R_{0} / R=1$.

2. Since $H=\left(F, \mathscr{L}_{0}\right)$ and since by $\mathrm{A},(F) \Rightarrow(J), H$ is maximal self orthogonal in $\mathscr{L}_{0}$ with respect to $l_{0}^{\prime}$ from which, as in I above, we conclude that $R_{0} \subset H, R_{0} \neq H$.

3. Let $l^{\prime}$ be a fixed nonzero element in $\mathscr{L}_{0}^{\perp}$. Then we have for all $l_{0}$ in $\mathscr{L}_{0}$ : $-\left(\operatorname{ad} l_{0}\right)^{\prime} \bar{l}^{\prime}=\alpha\left(l_{0}\right) \bar{l}^{\prime}$, where $\alpha$ is some linear form on $\mathscr{L}_{0}$. From this we conclude that $\delta\left(\exp l_{0}\right) \bar{l}^{\prime}=\left[\exp \alpha\left(l_{0}\right)\right] \bar{l}^{\prime}\left(l_{0} \in \mathscr{L}_{0}\right)$.

4. To obtain the proof of the sufficiency, it suffices to show the existence of an element $r$ in $R_{0} \cong H$ satisfying $\delta(\exp \operatorname{tr}) l_{0}^{\prime}=l_{0}^{\prime}+t \bar{l}^{\prime}$ for all real $t$. In fact, we observe first that the dual of $\mathscr{L}_{0}$ can be canonically identified with the factor space of $\mathscr{L}^{\prime}$ according to $\mathscr{L}_{0}^{\perp}$. We denote by $\pi$ the projection from $\mathscr{L}^{\prime}$ onto $\mathscr{L}_{0}^{\prime}$. Writing $\delta_{0}$ for the representation, corresponding to $\delta$, of $\overline{\mathscr{L}}_{0}$ on $\mathscr{L}_{0}^{\prime}$ one verifies easily that $\delta_{0}\left(\exp l_{0}\right) \pi=\pi \delta\left(\exp l_{0}\right)$ for all $l_{0}$ in $\mathscr{L}_{0}$. Since $H=\left(F, \mathscr{L}_{0}\right)$, taking into account that the orthogonal complement of $H$ in $\mathscr{L}_{0}^{\prime}$ is the image of $H^{\perp}$ under $\pi$, for any given $h^{\perp}$ in $H^{\perp}$ there exists an element $h_{0}$ in $H$ such that $\delta_{0}\left(\bar{h}_{0}\right) \pi l_{0}^{\prime}=\pi l_{0}^{\prime}+\pi h^{\perp}$, which gives $\delta\left(\bar{h}_{0}\right) l_{0}^{\prime}=l_{0}^{\prime}+h^{\perp}+c \bar{l}^{\prime}$, where $c$ is some real number. This gives (cf. 2) for any real $t$ :

$$
\delta\left(\bar{h}_{0} \exp (t r)\right) l_{0}^{\prime}=\delta\left(\bar{h}_{0}\right)\left(l_{0}^{\prime}+t \bar{l}^{\prime}\right)=l_{0}^{\prime}+h^{\perp}+\left[t \exp \left(\alpha\left(h_{0}\right)\right)+c\right] \bar{l}^{\prime} .
$$


Choosing $t=-c \exp \left(-\alpha\left(h_{0}\right)\right)$ and writing $\bar{h}=\bar{h}_{0} \exp (t r) \in \bar{H}$ this yields $\delta(\bar{h}) l_{0}^{\prime}$ $=l_{0}^{\prime}+h^{\perp}$, and since $h^{\perp}$ was arbitrary in $H^{\perp}$, we have the desired conclusion.

5. $R_{0}$ being the stabilizer of the restriction of $l_{0}^{\prime}$ to $\mathscr{L}_{0}$, we have $-(\operatorname{ad} r)^{\prime} l_{0}^{\prime}=\gamma(r) \bar{l}^{\prime}$ for all $r$ in $R_{0}$ where the linear form $\gamma$ vanishes if and only if $r$ belongs to $R$. On the other hand (cf. 3), $-(\operatorname{ad} r)^{\prime} \bar{l}^{\prime}=\alpha(r) \bar{l}^{\prime}\left(r \in R_{0}\right)$. From this, in view of 4, we conclude that it suffices to find an $r$ in $R_{0}$ such that $\gamma(r)=1$ and $\alpha(r)=0$. In fact, in this case $-(\operatorname{ad} r)^{\prime} l_{0}^{\prime}=\bar{l}^{\prime},-(\operatorname{ad} r)^{\prime} \bar{l}^{\prime}=0$, and thus $\delta(\exp t r) l^{\prime}=l_{0}^{\prime}+t \bar{l}^{\prime}$ for all real $t$.

6. Suppose now that $\mathscr{L}_{0}$ is an ideal; then evidently $\alpha \equiv 0$ and so the problem just mentioned has certainly solutions. Hence in this case $R \cong \mathscr{L}_{0}$ suffices to imply $H=(F)$, if $H=\left(F, \mathscr{L}_{0}\right)$ is valid.

7. Suppose that in the general case no $r$ with the required properties exists. Then there is a real number $d$ such that $\gamma(r)=d \alpha(r)$ for all $r$ in $R_{0}$ which gives $(\operatorname{ad} r)^{\prime}\left(l_{0}^{\prime}-d \bar{l}^{\prime}\right)=0$ identically and hence $R\left(l_{0}^{\prime}-d \bar{l}^{\prime}\right) \supseteqq R_{0}$. By the reasoning of 1 above, in this case $\operatorname{dim} R\left(l_{0}^{\prime}-d \bar{l}^{\prime}\right)=\operatorname{dim} R_{0}+1$, which excludes the equality sign. Since evidently $R\left(l_{0}^{\prime}-d \bar{l}^{\prime}\right) \cap \mathscr{L}_{0} \cong R_{0}, R\left(l_{0}^{\prime}-d \bar{l}^{\prime}\right)$ then cannot be contained in $\mathscr{L}_{0}$, and this contradiction proves Lemma 1. Q.E.D.

Lemma 2. Suppose that $\mathscr{L}_{0}$ is a subalgebra, containing $H$, of $\mathscr{L}$. Suppose also that $\operatorname{dim} \mathscr{L}_{0}+2=\operatorname{dim} \mathscr{L}$, and that $\mathscr{L} \mid \mathscr{L}_{0}$ is irreducible under the restriction of the adjoint representation of $\mathscr{L}$ to $\mathscr{L}_{0}$. Denote by $R_{0}$ the radical of the restriction of $l_{0}^{\prime}$ to $\mathscr{L}_{0}$. Then $H=\left(F, \mathscr{L}_{0}\right)$ implies $H=(F)$ if and only if $R \varsubsetneqq R_{0}$.

REMARK. In what follows we shall write $\mathscr{L} / \mathscr{L}_{0}=\operatorname{irr}\left(\mathscr{L}_{0}\right)$ to indicate that $\mathscr{L}_{0}$ has the properties of the first two sentences of Lemma 2.

Proof. I. To establish the necessity we proceed as in Lemma $1 . H=(F)$ implies (cf. A) $\operatorname{dim} H=\frac{1}{2}(\operatorname{dim} \mathscr{L}+\operatorname{dim} R), R \cong H$ and thus $R \cong R_{0}$. On the other hand $H=\left(F, \mathscr{L}_{0}\right)$ gives $\operatorname{dim} H=\frac{1}{2}\left(\operatorname{dim} \mathscr{L}_{0}+\operatorname{dim} R_{0}\right)$ and so finally $\operatorname{dim} R+2=\operatorname{dim} R_{0}$ and $R \varsubsetneqq R_{0}$.

II. 1. To prove the sufficiency, we observe again that $H=\left(F, \mathscr{L}_{0}\right)$ gives $R_{0} \subseteq H$. On the other hand, since

$$
\operatorname{dim} \mathscr{L}-\operatorname{dim} R=\operatorname{dim} \mathscr{L}_{0}-\operatorname{dim} R_{0}+2 k \quad(k=0,1,2),
$$

$R \varsubsetneqq R_{0}$ implies $\operatorname{dim} R+2=\operatorname{dim} R_{0}$.

2. We conclude from $\mathscr{L} / \mathscr{L}_{0}=\operatorname{irr}\left(\mathscr{L}_{0}\right)$, that there exists a pair of linearly independent elements $\left\{l_{1}^{\prime}, l_{2}^{\prime}\right\}$, orthogonal to $\mathscr{L}_{0}$, of $\mathscr{L}^{\prime}$, such that for all $l_{0}$ in $\mathscr{L}_{0}$

$$
\begin{aligned}
& -\left(\operatorname{ad} l_{0}\right)^{\prime} l_{1}^{\prime}=\delta\left(l_{0}\right)\left(l_{1}^{\prime}-\alpha l_{2}^{\prime}\right), \\
& -\left(\operatorname{ad} l_{0}\right)^{\prime} l_{2}^{\prime}=\delta\left(l_{0}\right)\left(\alpha l_{1}^{\prime}+l_{2}^{\prime}\right)
\end{aligned}
$$

where the linear form $\delta$ on $\mathscr{L}_{0}$ is not identically 0 and $\alpha \neq 0$. This gives also

$$
\begin{aligned}
& \delta\left(\exp l_{0}\right) l_{1}^{\prime}=\exp \delta\left(l_{0}\right)\left(\cos \left[\alpha \delta\left(l_{0}\right)\right] l_{1}^{\prime}+\sin \left[\alpha \delta\left(l_{0}\right)\right] l_{2}^{\prime}\right), \\
& \delta\left(\exp l_{0}\right) l_{2}^{\prime}=\exp \delta\left(l_{0}\right)\left(-\sin \left[\alpha \delta\left(l_{0}\right)\right] l_{1}^{\prime}+\cos \left[\alpha \delta\left(l_{0}\right)\right] l_{2}^{\prime}\right) .
\end{aligned}
$$


3. Next we observe that to prove $H=(F)$, it suffices to find a pair of elements $r_{1}, r_{2}$ in $R_{0}$ satisfying $\delta\left(\exp \left(t_{1} r_{1}+t_{2} r_{2}\right)\right) l_{0}^{\prime}=l_{0}^{\prime}+t_{1} l_{1}^{\prime}+t_{2} l_{2}^{\prime}$ for all real $t_{1}$ and $t_{2}$. In fact, reasoning as in 4 of part II of the proof of Lemma 1 , using $H=\left(F, \mathscr{L}_{0}\right)$ we conclude that for any given $h^{\perp}$ in $H^{\perp}$ there exists an element $\bar{h}_{0}$ in $\bar{H}$, and real numbers $c_{1}$ and $c_{2}$ such that $\delta\left(\bar{h}_{0}\right) l_{0}^{\prime}=l_{0}^{\prime}+h^{\perp}+c_{1} l_{1}^{\prime}+c_{2} l_{2}^{\prime}$. This gives

$$
\delta\left(\bar{h}_{0} \exp \left(t_{1} r_{1}+t_{2} r_{2}\right)\right) l_{0}^{\prime}=\delta\left(\bar{h}_{0}\right)\left(l_{0}^{\prime}+t_{1} l_{1}^{\prime}+t_{2} l_{2}^{\prime}\right)=l_{0}^{\prime}+h^{\perp}+t_{1}^{\prime} l_{1}^{\prime}+t_{2}^{\prime} l_{2}^{\prime},
$$

where

$$
\begin{aligned}
& t_{1}^{\prime}=c_{1}+\exp \left[\delta\left(l_{0}\right)\right]\left(\cos \left[\alpha \delta\left(h_{0}\right)\right] t_{1}-\sin \left[\alpha \delta\left(h_{0}\right)\right] t_{2}\right), \\
& t_{2}^{\prime}=c_{2}+\exp \left[\delta\left(l_{0}\right)\right]\left(\sin \left[\alpha \delta\left(h_{0}\right)\right] t_{1}+\cos \left[\alpha \delta\left(h_{0}\right)\right] t_{2}\right) .
\end{aligned}
$$

Choosing $t_{1}$ and $t_{2}$ such that $t_{1}^{\prime}=t_{2}^{\prime}=0$, and writing $\bar{h}=\bar{h}_{0} \exp \left(t_{1} r_{1}+t_{2} r_{2}\right)$, we obtain in this fashion $\delta(\bar{h}) l_{0}^{\prime}=l_{0}^{\prime}+h^{\perp}$. Since $\bar{h} \in \bar{H}$, in view of the arbitrariness of $h^{\perp} \in H^{\perp}$, this gives $H=(F)$.

4. We have for all $r$ in $R_{0}$ : $-(\operatorname{ad} r)^{\prime} l_{0}^{\prime}=\lambda_{1}(r) l_{1}^{\prime}+\lambda_{2}(r) l_{2}^{\prime}$, and we have $\lambda_{1}(r)=\lambda_{2}(r)=0$ if and only if $r$ belongs to $R$. Furthermore, since by 1 the codimension of $R$ in $R_{0}$ is $2, \lambda_{1}$ and $\lambda_{2}$ are linearly independent. By 3 , to prove $H=(F)$ it suffices to find a pair of elements $\left\{r_{1}, r_{2}\right\}$ in $R_{0}$ satisfying $\lambda_{j}\left(r_{i}\right)=\delta_{i j}$ and $\delta\left(r_{i}\right)=0(i, j=1,2)$. Such a pair certainly exists if the triple $\left\{\lambda_{1}, \lambda_{2}, \delta\right\}$ in $\mathscr{L}^{\prime}$ is linearly independent. In order to prove this we observe first that the system $\left\{l_{1}^{\prime}, l_{2}^{\prime}, l_{0}^{\prime}\right\}$ is linearly independent in $\mathscr{L}^{\prime}$. In fact, if this were not so, there would exist numbers $b_{1}$ and $b_{2}$ such that $l_{0}^{\prime}=b_{1} l_{1}^{\prime}+b_{2} l_{2}^{\prime}$. This would imply (cf. 2)

$$
\begin{aligned}
0 & =-(\operatorname{ad} r)^{\prime}\left(l_{0}^{\prime}-b_{1} l_{1}^{\prime}-b_{2} l_{2}^{\prime}\right) \\
& =\left[\lambda_{1}(r)-\delta(r)\left[b_{1}+\alpha b_{2}\right]\right] l_{1}^{\prime}+\left[\lambda_{2}(r)-\delta(r)\left[-\alpha b_{1}+b_{2}\right]\right] l_{2}^{\prime}
\end{aligned}
$$

for all $r$ in $R_{0}$, or $\lambda_{j}(r)=d_{j} \delta(r)(j=1,2)$, contradicting the linear independence of $\left\{\lambda_{1}, \lambda_{2}\right\}$. We denote by $W$ the subspace of $\mathscr{L}^{\prime}$ spanned by $\left\{l_{1}^{\prime}, l_{2}^{\prime}, l_{0}^{\prime}\right\}$. W is invariant under $(\mathrm{ad} r)^{\prime}\left(r \in R_{0}\right)$ and we have using the previous system as a basis

$$
\psi(r)=-\left.(\operatorname{ad} r)^{\prime}\right|_{W}=\left(\begin{array}{ccc}
\delta(r) & \alpha \delta(r) & \lambda_{1}(r) \\
-\alpha \delta(r) & \delta(r) & \lambda_{2}(r) \\
0 & 0 & 0
\end{array}\right)
$$

Let us write

$$
\left(x_{0}, x_{1}, x_{2}\right)=\left(\begin{array}{ccc}
x_{0} & \alpha x_{0} & x_{1} \\
-\alpha x_{0} & x_{0} & x_{2} \\
0 & 0 & 0
\end{array}\right)
$$

The collection $\hat{\mathscr{L}}$ of all matrices of this form is a Lie algebra with the basis $e_{0}=(1,0,0), e_{1}=(0,1,0), e_{2}=(0,0,1)$ satisfying $\left[e_{0}, e_{1}\right]=e_{1}-\alpha e_{2},\left[e_{0}, e_{2}\right]=\alpha e_{1}+e_{2}$, $\left[e_{1}, e_{2}\right]=0$. If the system $\left\{\lambda_{1}, \lambda_{2}, \delta\right\}$ is linearly dependent, we have $\delta=a_{1} \lambda_{1}+a_{2} \lambda_{2}$, and then the map $\psi$ defined above establishes a homomorphism of $R_{0}$ onto a 
2-dimensional subalgebra, different from $\left\{e_{1}, e_{2}\right\}$, of $\hat{\mathscr{L}}$. Therefore, a proof of the impossibility of this suffices to prove Lemma 2 . Let $\mathscr{L}_{1}$ be a subalgebra of $\hat{\mathscr{L}}$ with the basis

$$
f_{1}=a_{0} e_{0}+a_{1} e_{1}+a_{2} e_{2}, \quad f_{2}=b_{0} e_{0}+b_{1} e_{1}+b_{2} e_{2},
$$

where $a_{0} \neq 0$, say. Replacing, if necessary, $\left\{f_{1}, f_{2}\right\}$ by $\left\{\left(1 / a_{0}\right) f_{1}, f_{2}-\left(b_{0} / a_{0}\right) f_{1}\right\}$, we can assume $a_{0}=1, b_{0}=0$. But then we must have also $\left[f_{1}, f_{2}\right]=\left[e_{0}, f_{2}\right]=c f_{2}$ where $c$ is a real number, which is impossible, since the roots of ad $e_{0}$ are $1 \pm i \alpha$, and $\alpha \neq 0$.

Lemma 3. Suppose that $H=(G)$ and that $\mathscr{L}_{0}$ is a subalgebra of $\mathscr{L}$ containing $H$. Then we have also $H=\left(G, \mathscr{L}_{0}\right)$.

Proof. We observe again that the dual of $\mathscr{L}_{0}$ can be canonically identified with the quotient space of $\mathscr{L}^{\prime}$ according to $\mathscr{L}_{0}^{\perp}$, and that the projection $\pi$ of $\mathscr{L}^{\prime}$ onto $\mathscr{L}_{0}^{\prime}$, consisting in restriction to $\mathscr{L}_{0}$, maps $H^{\perp}$ onto the orthogonal complement of $H$ in $\mathscr{L}_{0}^{\prime}$. On the other hand (cf. $\left.2 \mathrm{a}, \mathrm{b}\right) H=(G)$ means simply that $H$ is maximal self orthogonal with respect to $l_{0}^{\prime}+h^{\perp}$ for all $h^{\perp}$ in $H^{\perp}$; but then it is certainly maximal self orthogonal in $\mathscr{L}_{0}$ with respect to all $\pi\left(l_{0}^{\prime}+h^{\perp}\right)$.

To finish the proof of the statement that $H=(G)$ implies $H=(F)$, we observe first that if $\mathscr{L}_{1}$ is a subalgebra of $\mathscr{L}$ such that

$$
\operatorname{dim} \mathscr{L}>\operatorname{dim} \mathscr{L}_{1}+1 \text { and } \mathscr{L} \mid \mathscr{L}_{1} \neq \operatorname{irr}\left(\mathscr{L}_{1}\right)
$$

(cf. Remark after Lemma 2), then there exists a subalgebra $\mathscr{L}_{2}$ containing $\mathscr{L}_{1}$ such that if $\operatorname{dim} \mathscr{L}_{2}>\operatorname{dim} \mathscr{L}_{1}+1$, we have $\mathscr{L}_{2} / \mathscr{L}_{1}=\operatorname{irr}\left(\mathscr{L}_{1}\right)$ and $\operatorname{dim} \mathscr{L}_{2}=\operatorname{dim} \mathscr{L}_{1}+2$; in fact, this follows from an easy application of the theorem of Lie. Therefore, by virtue of Lemmas $1-3$, it suffices to prove that if $H=(G)$, and if $\mathscr{L}_{0}$ is a subalgebra of codimension 1 or 2 of $\mathscr{L}_{1}$ such that in the latter case $\mathscr{L} / \mathscr{L}_{0}=\operatorname{irr}\left(\mathscr{L}_{0}\right)$, then $\mathscr{L}_{0}$ satisfies the conditions of Lemmas 1-2. Indeed, observing that trivially $H=(F, H)$, we can finish then our proof by induction, using an increasing sequence of appropriately chosen subalgebras containing $H$. By arguments used repeatedly, $H=(G)$ implies that $H$ is maximal self orthogonal with respect to $l_{0}^{\prime}+l_{0}^{\perp}\left(l_{0}^{\frac{1}{1}} \in \mathscr{L}_{0}^{\frac{1}{1}}\right)$; hence $R\left(l_{0}^{\prime}+l_{0}^{\perp}\right) \subseteq R_{0} \subseteq H \subseteq \mathscr{L}_{0} \subset \mathscr{L}$. Also,

$$
\operatorname{dim} H=\frac{1}{2}(\operatorname{dim} \mathscr{L}+\operatorname{dim} R)=\frac{1}{2}\left(\operatorname{dim} \mathscr{L}_{0}+\operatorname{dim} R_{0}\right)
$$

and therefore $\operatorname{dim} R_{0}-\operatorname{dim} R=\operatorname{dim} \mathscr{L}-\operatorname{dim} \mathscr{L}_{0}=1$ or 2 ; hence always positive.

For later use we list here the following two statements which are immediate consequences of Lemmas 1-2 resp. and of the equivalence of properties $F$ and $J$ :

LEMmA $1^{\prime}$. Let $\mathscr{L}_{0}$ be a subalgebra, of codimension one, of $\mathscr{L}$, containing $H$, and suppose that $H=\left(J, \mathscr{L}_{0}\right)$. Then $H=(J)$ if and only if for all $l_{0}^{\perp}$ in $\mathscr{L}_{0}^{\perp} R\left(l_{0}^{\prime}+l_{0}^{\perp}\right)$ is contained in $\mathscr{L}_{0}$. If $\mathscr{L}_{0}$ is an ideal, the last condition follows from $R\left(l_{0}^{\prime}\right) \subseteq \mathscr{L}_{0}$.

Lemma 2 '. Suppose that the subalgebra $\mathscr{L}_{0}$ of codimension 2 in $\mathscr{L}$ contains $H$ and that $\mathscr{L} \mid \mathscr{L}_{0}=\operatorname{irr}\left(\mathscr{L}_{0}\right)$. Then $H=\left(J, \mathscr{L}_{0}\right)$ implies $H=(J)$ if and only if $R \subset R_{0}$, $R \neq R_{0}$, where $R$ and $R_{0}$ are the radicals of $l_{0}^{\prime}$ in $\mathscr{L}$ and $\mathscr{L}_{0}$ resp. 
4. Throughout this section, as in $\S 3$, we keep the exponential algebra $\mathscr{L}$, and the nonzero element $l_{0}^{\prime}$ of $\mathscr{L}^{\prime}$ fixed. We recall (cf. 1), that if the subalgebra $H$ is subordinated to $l_{0}^{\prime}$, ind $\left(H, l_{0}^{\prime}\right)$ denotes the unitary representation of $\overline{\mathscr{L}}$ induced by the character $\chi(\bar{h})=\exp \left(i\left(h, l_{0}^{\prime}\right)\right)$ of $\bar{H}(h \in H)$.

One part of the proof of the following Proposition follows closely certain reasonings of Kirillov (cf. [3, particularly Lemma 5.1]) and Bernat (cf. [1, Chapitre III]).

Proposition 2. Suppose that the subalgebra $H$ of $\mathscr{L}$ is subordinated to $l_{0}^{\prime}$. Then the unitary representation ind $\left(H, l_{0}^{\prime}\right)$ of $\overline{\mathscr{L}}$ is irreducible if and only if $H=(J)$.

Proof. We shall use induction according to the dimension of the groups considered. The Proposition being trivially true in the 1-dimensional case, in what follows we shall suppose $\operatorname{dim} \mathscr{L}>1$, and assume its validity for groups of dimension less than $\operatorname{dim} \mathscr{L}$. We divide the proof into three parts.

A. We suppose first, that there exists a nonzero ideal $I$, orthogonal to $l_{0}^{\prime}$ in $\mathscr{L}$; in what follows we denote by the same letter a minimal ideal having this property.

1. Let us prove that if either $H=(J)$, or ind $\left(H, l^{\prime}\right)=$ irreducible, we have $I \subseteq H$.

$\alpha$. We know that if $H=(J), H$ as a subspace of $\mathscr{L}$ is maximal self orthogonal with respect to $l_{0}^{\prime}$. Therefore, it suffices to verify that the subalgebra $H_{1}=H+I$ is self orthogonal. But this is clear since $\left[H_{1}, H_{1}\right]=[H, H]+I$, and by virtue of $H<l_{0}^{\prime}$, $[H, H]$ is orthogonal to $l_{0}^{\prime}$.

$\beta$. Suppose now, that ind $\left(H, l_{0}^{\prime}\right)$ is irreducible. If $\mathscr{L}_{0}$ is a subalgebra, containing $H$, of $\mathscr{L}$, we write ind $\left(H, l_{0}^{\prime} ; \mathscr{L}_{0}\right)$ for the unitary representation induced in $\overline{\mathscr{L}}_{0}$ by the character of $\bar{H}$ corresponding to $l_{0}^{\prime}$. We assume that $I$ is not contained in $H$, and prove that then ind $\left(H, l_{0}^{\prime} ; H+I\right)$ is reducible. Since the representation induced in $\overline{\mathscr{L}}$ by the representation ind $\left(H, l_{0}^{\prime} ; H+I\right)$ of $\exp (H+I)$ is the same as ind $\left(H, l_{0}^{\prime}\right)$, and since the reducibility of the inducing representation implies that of the induced one in this fashion we shall obtain a contradiction. Observe that if $I \notin H$, then, by virtue of the minimality of $I$, we have $H \cap I=0$. We distinguish two cases.

(a) If $[H, I]=0, \exp (H+I)$ is isomorphic to the direct product of $\bar{H}$ and $\bar{I}$ and in this case ind $\left(H, l_{0}^{\prime} ; H+I\right)$ is clearly reducible.

(b) Suppose now that $[H, I] \neq 0$. We have either

$$
\operatorname{dim} I=1, I=\{e\} \text { and } \operatorname{ad} h(e)=\lambda(h) e,
$$

or $\operatorname{dim} I=2, I=\left\{l_{1}, l_{2}\right\}$ and

$$
\text { ad } h\left(l_{1}\right)=\delta(h)\left(l_{1}-\alpha l_{2}\right), \quad \text { ad } h\left(l_{2}\right)=\delta(h)\left(\alpha l_{1}+l_{2}\right),\left[l_{1}, l_{2}\right]=0,
$$

where $\lambda$ and $\delta$ are nontrivial 1-dimensional representations of $H$, and $\alpha \neq 0$. We consider here in detail the second case only; the first can be settled in a similar fashion. We denote by $H_{\delta}$ the kernel of $\delta$, and by $H_{0}$ the subspace of elements, orthogonal to $l_{0}^{\prime}$, of $H$. Observe that $H_{0}=H$ is impossible; in fact in this case, by virtue of the irreducibility of ind $\left(H, l_{0}^{\prime}\right)$ we would have $H=\mathscr{L}$ and hence $l_{0}^{\prime}=0$ 
which we excluded at the beginning of this section. If $H_{\delta}=H_{0}, H+I / H_{0}$ is isomorphic to the algebra $\hat{\mathscr{L}}=\left\{f_{0}, f_{1}, f_{2}\right\}$ with $\left[f_{0}, f_{1}\right]=f_{1}-\alpha f_{2},\left[f_{0}, f_{2}\right]=\alpha f_{1}+f_{2}$, and we obtain ind $\left(H, l_{0}^{\prime} ; H+I\right)$ by lifting up to $\exp (H+I)$ a representation, induced by a character of $\exp \left\{f_{0}\right\}$, of $\exp \hat{\mathscr{L}}$, but this is always reducible. If $S=H_{0} \cap H_{\delta}$ is properly contained in $H_{\delta}$, we can write $H=X+Y+S$, where $X=\{x\}, Y=\{y\}$ with $\delta(x)=0$ and $\delta(y)=1,\left(y, l_{0}^{\prime}\right)=0$ resp. $H<l_{0}^{\prime}$ implies $[H, H] \subseteq S$; hence $S$ is an ideal in $H+I . H+I / S$ is isomorphic to the direct product of $\hat{\mathscr{L}}$ with a 1-dimensional algebra $R$, and ind $\left(H, l_{0}^{\prime} ; H+I\right)$ is obtained by lifting up to exp $(H+I)$ a representation of $\exp (\hat{\mathscr{L}} \times R)$ induced by a character of $\bar{R}$, which is again reducible.

2. For what follows observe that the dual of $\tilde{\mathscr{L}}=\mathscr{L} \mid I$ can be canonically identified to the subspace $I^{\perp}$ of $\mathscr{L}^{\prime}$, and that by virtue of our assumptions we have $l_{0}^{\prime} \in I^{\perp}$.

Lemma 4. Suppose that the subalgebra $H$ of $\mathscr{L}$ is subordinated to the element $l_{0}^{\prime}$ of $\mathscr{L}^{\prime}$, and that $H$ contains the ideal $I$, orthogonal to $l_{0}^{\prime}$, in $\mathscr{L}$. We denote by $\tilde{H}$ the canonical image of $H$ in $\tilde{\mathscr{L}}=\mathscr{L} \mid I$. Then we have $H=(J)$ if and only if $\tilde{H}=(J)$.

Proof. We denote by $\tilde{\delta}$ the representation corresponding to $\delta$ of $\exp \tilde{\mathscr{L}}$ on $\tilde{\mathscr{L}}^{\prime}$. Writing $l$ for the image in $\tilde{\mathscr{L}}$ of $l \in \mathscr{L}$, we have with the identification explained above $\tilde{\delta}(\bar{l}) l_{0}^{\prime}=\delta(\bar{l}) l_{0}^{\prime}$; hence the orbits of $l_{0}^{\prime}$ with respect to $\delta$ and $\tilde{\delta}$ coincide. Since also $l_{0}^{\prime}+\tilde{H}^{-}=l_{0}^{\prime}+H^{\perp}$, to finish the proof it suffices to observe that, by virtue of $I \subset H, \operatorname{dim} \tilde{H}+\operatorname{dim} I=\operatorname{dim} H$, and therefore $\operatorname{dim} \tilde{H}=\operatorname{dim} \tilde{\mathscr{L}}-\frac{1}{2} \operatorname{dim} O$ is equivalent to $\operatorname{dim} H=\operatorname{dim} \mathscr{L}-\frac{1}{2} \operatorname{dim} O$.

3. We denote by $\rho$ the canonical homomorphism of $\overline{\mathscr{L}}$ onto exp $\tilde{\mathscr{L}}$; we have ind $\left(H, l_{0}^{\prime}\right)=$ ind $\left(\tilde{H}, l_{0}^{\prime}\right) \cdot \rho$. To finish the proof of Proposition 2 in case A, suppose first, that ind $\left(H, l_{0}^{\prime}\right)$ is irreducible. Then so is ind $\left(\tilde{H}, l_{0}^{\prime}\right)$; hence by virtue of the hypothesis of the induction, we have $\tilde{H}=(J)$ which by Lemma 4 implies $H=(J)$. Conversely, $H=(J)$ gives $\tilde{H}=(J)$; hence by induction ind $\left(\tilde{H}, l_{0}^{\prime}\right)$ and along with it ind $\left(H, l_{0}^{\prime}\right)$ is irreducible.

B. Here we suppose that the orthogonal complement of $l_{0}^{\prime}$ does not contain any nontrivial ideal but that there exists a minimal ideal $I$ not contained in the center $C$ of $\mathscr{L}$. If $\operatorname{dim} I=1$, and $I=\{e\}$, we have ad $l(e)=\lambda(l) e(l \in \mathscr{L})$, where $\lambda \not \equiv 0$, since $I$ is not contained in $C$. Also, $\left(e, l_{0}^{\prime}\right)$ is nonzero since otherwise we would have case A. If $\operatorname{dim} I=2$, we can choose a basis $\left\{l_{1}, l_{2}\right\}$ in $I$, such that

$$
\text { ad } l\left(l_{1}\right)=\delta(l)\left(l_{1}-\alpha l_{2}\right), \text { ad } l\left(l_{2}\right)=\delta(l)\left(\alpha l_{1}+l_{2}\right) \quad(l \in \mathscr{L} ; \alpha \neq 0),
$$

where as before $\delta \not \equiv 0$ and $\left(l_{1}, l_{0}^{\prime}\right)^{2}+\left(l_{2}, l_{0}^{\prime}\right)^{2} \neq 0$.

We denote by $\mathscr{L}_{0}$ the kernel of $\lambda$ or of $\delta$ resp.

1. We prove first, that if either $H=(J)$ or ind $\left(H, l_{0}^{\prime}\right)$ is irreducible, $H$ is contained in $\mathscr{L}_{0}$. We shall do this by assuming that $H \nsubseteq \mathscr{L}_{0}$ and by showing that this leads to a contradiction. We give the proof in three steps.

a. Let us show that if $H<l_{0}^{\prime}$ and $H \neq \mathscr{L}_{0}$, then $H \cap I=0$. We take the case when $\operatorname{dim} I=2$; the other case can be settled similarly. Since $I$ is a minimal ideal, if 
$H \cap I \neq 0$, we have $I \subset H$. Since, by virtue of our assumption, $H$ is not contained in $\mathscr{L}_{0}$, there exists an element $h$ in $H$ with $\delta(h)=1$. We have then, since $H<l_{0}^{\prime}$ :

$$
\begin{aligned}
& 0=\left(\left[h, l_{1}\right], l_{0}^{\prime}\right)=\left(l_{1}, l_{0}^{\prime}\right)-\alpha\left(l_{2}, l_{0}^{\prime}\right), \\
& 0=\left(\left[h, l_{2}\right], l_{0}^{\prime}\right)=\alpha\left(l_{1}, l_{0}^{\prime}\right)+\left(l_{2}, l_{0}^{\prime}\right)
\end{aligned}
$$

which gives $\left(l_{j}, l_{0}^{\prime}\right)=0(j=1,2)$, contradicting the assumption that $I$ is not orthogonal to $l_{0}^{\prime}$.

b. Suppose now that the representation ind $\left(H, l_{0}^{\prime}\right)$ is irreducible. We observe that $H \notin \mathscr{L}_{0}$ implies $[H, I] \neq 0$. Since by virtue of $H \cap I=0$, a word by word repetition of the argument of A.l. $\beta$.b. shows that ind $\left(H, l_{0}^{\prime} ; H+I\right)$ is reducible, contradicting the irreducibility of ind $\left(H, l_{0}^{\prime}\right)$. Hence, in this case $H \subset \mathscr{L}_{0}$.

c. Next we consider the case when $H=(J)$. Then by virtue of Proposition 1 and Lemma 3, also $H=(J, H+I)$. We shall show that this is impossible, if $H \cap I=0$. Here we shall restrict ourselves to the discussion of the case $\operatorname{dim} I=1$; the case of an $I$ with $\operatorname{dim} I=2$ can be settled similarly (cf. for this also A.l.ß.b.). We denote again by $H_{0}$ the subspace of elements, orthogonal to $l_{0}^{\prime}$ of $H$, and $H_{\lambda}$ the intersection of $H$ and of the kernel of $\lambda$; we have then $H_{\lambda} \varsubsetneqq H$. If $H_{\lambda} \cap H_{0}=H_{\lambda}$, the factor algebra of $H+I$ according to $H_{\lambda}$ is isomorphic to $\hat{\mathscr{L}}=\left\{J_{0}, J_{1}\right\}$, with $\left[J_{0}, J_{1}\right]=J_{1}$, the image of $H$ being $\left\{J_{0}\right\}$. Then by Lemma $4,\left\{J_{0}\right\}$ must have the property $J$ in $\hat{\mathscr{L}}$ with respect to an element $J^{\prime}$ of $\hat{\mathscr{L}}^{\prime}$ with $\left(J_{1}, J_{0}^{\prime}\right) \neq 0$ (since $\left.\left(e, l_{0}^{\prime}\right) \neq^{\prime} 0\right)$, which can be shown to be impossible by a simple computation. If $S=H_{\lambda} \cap H_{0}$ is properly contained in $H_{\lambda}$, we can write $H=X+Y+S, X=\{x\}, \quad Y=\{y\}$ with $\lambda(x)=0, \lambda(y)=1$ and $\left(J, l_{0}^{\prime}\right)=0$. Since $H<l_{0}^{\prime}$, we have $[H, H] \subset[S, S]$, from which we conclude that $S$ is an ideal in $H+I$. The factor algebra of $H+I$ according to $S$ is isomorphic to $\hat{\mathscr{L}}_{1}=\left\{J_{0}, J_{1}, J_{2}\right\}$, where $\left[J_{0}, J_{1}\right]=J_{1}$ and $\left[J_{0}, J_{2}\right]=\left[J_{1}, J_{2}\right]=0$; the image of $H$ is $\left\{J_{0}, J_{2}\right\}$. Again an application of Lemma 4 shows that $\left\{J_{0}, J_{2}\right\}$ must have the property in $\hat{\mathscr{L}}_{1}$ with respect to $J^{\prime} \in \hat{\mathscr{L}}^{\prime}$, satisfying $\left(J_{1}, J^{\prime}\right) \neq 0,\left(J_{0}, J^{\prime}\right)=0$, which can easily be disproved.

Summing up, if either ind $\left(H, l_{0}^{\prime}\right)$ is irreducible, or $H$ has the property $J, H$ must be contained in the centralizer of $I$.

2. We suppose now that ind $\left(H, l_{0}^{\prime}\right)$ is irreducible, and prove, that this implies $H=(J) . H$ being contained in $\mathscr{L}_{0}$, we can form the representation ind $\left(H, l_{0}^{\prime} ; \mathscr{L}_{0}\right)$ which, too, is irreducible and therefore, by the hypothesis of our induction, $H=\left(J, \mathscr{L}_{0}\right)$. Hence to finish the proof, by virtue of Lemma $1^{\prime}$ it suffices to show, that the radical $R$ of $l_{0}^{\prime}$ is contained in the ideal $\mathscr{L}_{0}$. If this is not so then, in the case of an $I$ with $\operatorname{dim} I=2$, there exists an element $r$ in $R$ satisfying $\delta(r)=1$, which gives

$$
\begin{aligned}
& 0=\left(\left[r, l_{1}\right], l_{0}^{\prime}\right)=\left(l_{1}, l_{0}^{\prime}\right)-\alpha\left(l_{2}, l_{0}^{\prime}\right), \\
& 0=\left(\left[r, l_{2}\right], l_{0}^{\prime}\right)=\alpha\left(l_{1}, l_{0}^{\prime}\right)+\left(l_{2}, l_{0}^{\prime}\right)
\end{aligned}
$$

implying that $I$ is orthogonal to $l_{0}^{\prime}$. The case $\operatorname{dim} I=1$ can be settled similarly.

3. Suppose finally that $H=(J)$. Then also $H=\left(J, \mathscr{L}_{0}\right)$ and therefore by induction, ind $\left(H, l_{0}^{\prime} ; \mathscr{L}_{0}\right)$ is irreducible. We denote this representation by $T$ and show that $T$ 
induces an irreducible representation in $\overline{\mathscr{L}}$. Since this is precisely ind $\left(H, l_{0}^{\prime}\right)$ in this fashion we prove also that $H=(J)$ implies the irreducibility of ind $\left(H, l_{0}^{\prime}\right)$. To obtain the desired conclusion, by results of Mackey [4], it suffices to observe that

(a) the orbits of the representation induced by $\delta$ on $I^{\prime}=\mathscr{L}^{\prime} / I^{\perp}$ are countably separated and simply connected (cf. [9, p. 156]); hence

(b) the stabilizer of the image $\pi l_{0}^{\prime}$ of $l_{0}^{\prime}$ in $I^{\prime}$ is connected, and therefore coincides with $\overline{\mathscr{L}}_{0}$,

(c) the ideal $I$ being in the center of $\mathscr{L}_{0}$, the restriction of $T$ to $\bar{I}$ is unit operator times the character $\chi$ of $\bar{I}$ corresponding to $\pi l_{0}^{\prime}$; that is $\chi(\exp (t e))=\exp \left[i t\left(e, l_{0}^{\prime}\right)\right]$, if $\operatorname{dim} I=1$, and $\chi\left(\exp \left(t_{1} l_{1}+t_{2} l_{2}\right)\right)=\exp \left[i\left(t_{1} l_{1}+t_{2} l_{2}, l_{0}^{\prime}\right)\right]$, if $\operatorname{dim} I=2$.

d. We continue to denote by $C$ the center of $\mathscr{L}$. If we do not have case $\mathrm{A}$, then $\operatorname{dim} C=0$, or 1 . The former possibility implying $\mathrm{B}$, it remains to consider the case when $\operatorname{dim} C=1, C=\{c\}$ and $\left(c, l_{0}^{\prime}\right) \neq 0$. Then there exists an ideal $I$, containing $C$, such that $\operatorname{dim} I / C=1$ or $\operatorname{dim} I / C=2$. In the first case we write $I=\left\{l_{0}, c\right\}$, ad $l\left(l_{0}\right)=\delta(l) l_{0}+\gamma(l) c(l \in \mathscr{L})$; observe that we can suppose $\left(l_{0}, l_{0}^{\prime}\right)=0$. We denote by $\mathscr{L}_{0}$ the kernel of $\gamma$; one sees easily that this is the stabilizer in $\mathscr{L}$ of the image $\pi l_{0}^{\prime}$ in $I^{\prime}$ of $l_{0}^{\prime}$ under the representation induced in $I^{\prime}$ by $d \delta(l)=-(\operatorname{ad} l)^{\prime}(l \in \mathscr{L})$. If $\operatorname{dim} I / C=2$ we can assume

$$
\begin{aligned}
I & =\left\{l_{1}, l_{2}, c\right\}, \text { ad } l\left(l_{1}\right)=\delta(l)\left(l_{1}+\alpha l_{2}\right)+\gamma_{1}(l) c, \\
\operatorname{ad} l\left(l_{2}\right) & =\delta(l)\left(-\alpha l_{1}+l_{2}\right)+\gamma_{2}(l) c \quad(l \in \mathscr{L}),
\end{aligned}
$$

where $\delta \not \equiv 0$ and $\left(l_{j}, l_{0}\right)=0(j=1,2)$. In this case, too, $I$ is abelian (cf. [9, p. 155]). In fact, to this end it suffices to observe that for all $l$ in $\mathscr{L}$

$$
\operatorname{ad} l\left(\left[l_{1}, l_{2}\right]\right)=\left[\operatorname{ad} l\left(l_{1}\right), l_{2}\right]+\left[l_{1}, \operatorname{ad} l\left(l_{2}\right)\right]=2 \delta(l)\left[l_{1}, l_{2}\right],
$$

hence, since $\delta \not \equiv 0$, we have A or B. We denote by $\mathscr{L}_{0}$ the intersection of the kernels of $\gamma_{1}$ and $\gamma_{2}$, and observe, that it can be interpreted as a stabilizer exactly as above. We divide the discussion in two major parts.

1. First we show that if $H$ is a subalgebra not contained in $\mathscr{L}_{0}$ in either of the above cases, of $\mathscr{L}$, such that either ind $\left(H, l_{0}^{\prime}\right)$ is irreducible, or $H=(J)$, then there exists a subalgebra $H_{1}$ contained in $\mathscr{L}_{0}$ such that ind $\left(H, l_{0}^{\prime}\right)=$ ind $\left(H_{1}, l_{0}^{\prime}\right)$ and that $H_{1}=(J)$ is equivalent to $H=(J)$. We write $\hat{H}=H+I$ and observe, that it suffices to find a subalgebra $H_{1}$ of $\hat{H} \cap \mathscr{L}_{0}$, such that

$$
\text { ind }\left(H, l_{0}^{\prime}, \hat{H}\right)=\text { ind }\left(H_{1}, l_{0}^{\prime}, \hat{H}\right) \quad \text { and } \quad H=(J, \hat{H}), H_{1}=(J, \hat{H}) \text {. }
$$

In fact, we have then certainly ind $\left(H, l_{0}^{\prime}\right)=$ ind $\left(H_{1}, l_{0}^{\prime}\right)$. Suppose now, that $H=(J)$, say. To prove, that this implies $H_{1}=(J)$, we can proceed, as at the end of the proof of Proposition 1. In fact, if the subalgebras $\mathscr{L}_{1}$ and $\mathscr{L}_{2}$ are such, that $\hat{H} \subseteq \mathscr{L}_{1} \subset \mathscr{L}_{2}$ and that either $\operatorname{dim} \mathscr{L}_{1}+1=\operatorname{dim} \mathscr{L}_{2}$, or $\operatorname{dim} \mathscr{L}_{1}+2=\operatorname{dim} \mathscr{L}_{2}$ and $\mathscr{L}_{2} / \mathscr{L}_{1}=\operatorname{irr}\left(\mathscr{L}_{1}\right)$, then using $H=(J)$ we conclude, that $\mathscr{L}_{1}$ satisfies, with respect to $\mathscr{L}_{2}$, the conditions of Lemma $1^{\prime}$ or Lemma $2^{\prime}$ on $\mathscr{L}_{0}$, necessary that $H_{1}=\left(J, \mathscr{L}_{1}\right)$ imply $H_{1}=\left(J, \mathscr{L}_{2}\right)$. Starting with $H_{1}=(J, \hat{H})$, we can then finish the proof of $H_{1}=(J)$ 
by induction. Let us write $H_{y}=H \cap \mathscr{L}_{0}$; we are going to prove that $H_{1}=H_{y}+I$ has the required properties. We begin by observing that, since $I$ is abelian, we have $H_{1} \subset \hat{H} \cap \mathscr{L}_{0}$. To prove, that $H_{1}<l_{0}^{\prime}$ it suffices to remark, that

$$
\left[H_{1}, H_{1}\right]=\left[H_{\gamma}, H_{\gamma}\right]+\left[H_{\gamma}, I\right]
$$

$H<l_{0}^{\prime}$ implies $\left[H_{y}, H_{\gamma}\right] \subset[H, H] \perp l_{0}^{\prime}$, and $\left[H_{\gamma}, I\right]$ is contained in $\left\{l_{0}\right\}$ or in $\left\{l_{1}, l_{2}\right\}$ resp.; hence is again orthogonal to $l_{0}^{\prime}$. If $H=(J)$, then $C \subset R\left(l_{0}^{\prime}\right) \subset H$, hence $C \subset H$. Similarly, if ind $\left(H, l_{0}^{\prime}\right)=$ irreducible, then $C \subset H$ (cf. A.1.ß.a.). We denote by $H_{\delta}$ the intersection of the kernel of $\delta$ and of $H$. Then $Z=H_{\delta} \cap H_{y}$ is the centralizer of $I$ in $H$, and hence it is an ideal in $H$. We denote by $S$ the subspace of elements, orthogonal to $l_{0}^{\prime}$, of $Z$; we have $Z=C+S$. Next we show, that $S$ is an ideal in $H$; since $[S, I]=0$, this implies, that it is an ideal in $\hat{H}=H+I$, too. But

$$
[S, H]=[S+C, H]=[Z, H] \subseteq Z=S+C,
$$

and thus, by virtue of $H<l_{0}^{\prime},[S, H] \subset S$, which proves our statement. Observe, that evidently $S \subset H_{1}$. Let $\phi$ denote the canonical homomorphism of $\hat{H}$ on its factor algebra $\tilde{\mathscr{L}}$ according to $S$. We write $\tilde{H}$ and $\tilde{H}_{1}$ for the images, under $\phi$ of $H$ and $H_{1}$ resp., and denote by $l_{0}^{\prime}$ the elements, corresponding to $l_{0}^{\prime}$, of $\tilde{\mathscr{L}}^{\prime}$; evidently $\tilde{H}, \tilde{H}_{1}<l_{0}^{\prime}$. In order to have ind $\left(H, l_{0}^{\prime} ; \hat{H}\right)=$ ind $\left(H_{1}, l_{0}^{\prime} ; \hat{H}\right)$; by known properties of induced representations it suffices to show ind $\left(\tilde{H}, \tilde{l}_{0}^{\prime}\right)=$ ind $\left(\tilde{H}_{1}, \tilde{l}_{0}^{\prime}\right)$. Similarly, using Lemma 4 we conclude, that $\tilde{H}=(J)$ implies $H=(J)$, and $\tilde{H}_{1}=(J)$ implies $H_{1}=(J)$. Next we turn to the discussion of the structure of $\tilde{\mathscr{L}}$; here we distinguish two cases.

$\alpha . \operatorname{dim} I / C=1$. With the notations introduced before, $I=\left\{l_{0}, e\right\}$ and

$$
\text { ad } l\left(l_{0}\right)=\delta(l) l_{0}+\gamma(l) c \quad(l \in \mathscr{L}),\left(l_{0}, l_{0}^{\prime}\right)=0 .
$$

Since we exclude $A$ and $B$, we must have $\gamma \not \equiv 0$, and thus, because of $H \notin \mathscr{L}_{0}$, $H_{\gamma} \varsubsetneqq H$. If $H_{\gamma}=H_{\delta}$, then we have on $\hat{H}: \gamma=d \delta$, where $d$ is a nonzero constant, and therefore ad $\hat{h}\left(l_{0}+d c\right)=\delta(\hat{h})\left(l_{0}+d c\right)$ for all $\hat{h}$ in $\hat{H}$, and $\left(l_{0}+d c_{1} l_{0}^{\prime}\right) \neq 0$. Hence, by B above if either ind $\left(H, l_{0}^{\prime} ; \hat{H}\right)$ is irreducible, or $H=(J, \hat{H}), H$ must be contained in $H_{\delta}+I=H_{\gamma}+I \subset \mathscr{L}_{0}$, contradicting $H \notin \mathscr{L}_{0}$. Observe, that $H<l_{0}^{\prime}$ excludes $l_{0} \in H$; indeed, otherwise we would have for all $h$ in $H: 0=\left(\left[h_{1} l_{0}\right], l_{0}^{\prime}\right)=\gamma(h)\left(c, l_{0}^{\prime}\right)$, and therefore, because of $\left(c, l_{0}^{\prime}\right) \neq 0, H \subset \mathscr{L}_{0}$. Summing up, we have to consider two subcases.

a. $H_{\delta}=H$. We can write $H=X+C+S$, where $X=\{x\}$ with $\left(x, l_{0}^{\prime}\right)=0$ and $\gamma(x)=1$. Thus we have $\tilde{\mathscr{L}}=\left\{e_{1}, e_{2}, e_{3}\right\}$, with the only nonvanishing bracket $\left[e_{1}, e_{2}\right]=e_{3}$, and $\left(e_{j}, \tilde{l}_{0}^{\prime}\right)=0(j=1,2),\left(e_{3}, \tilde{l}_{0}^{\prime}\right) \neq 0$, finally $\tilde{H}=\left\{e_{1}, e_{3}\right\}, \tilde{H}_{1}=\left\{e_{2}, e_{3}\right\}$.

b. $H_{\delta} \cap H_{\gamma} \subset H_{\gamma}, H_{\delta} \neq H_{\gamma}$. We have then $H=X+Y+C+S, X=\{x\}, Y=\{y\}$, $0=\left(x, l_{0}^{\prime}\right)=\left(y, l_{0}^{\prime}\right), \gamma(x)=1, \delta(x)=0, \gamma(y)=0, \delta(y)=1$. Observe, that $[x, y] \equiv x$ $(\bmod S)$; in fact, $H<l_{0}^{\prime}$ gives $[x, y] \equiv a x(\bmod S)$ and $a=\gamma([x, y])=1$. Therefore $\tilde{\mathscr{L}}=\left\{e_{0}, e_{1}, e_{2}, e_{3}\right\}$ with the nonvanishing brackets $\left[e_{0}, e_{1}\right]=-e_{1},\left[e_{0}, e_{2}\right]=e_{2}$, $\left[e_{1}, e_{2}\right]=e_{3}$. Furthermore $H=\left\{e_{0}, e_{2}, e_{3}\right\}, \tilde{H}_{1}=\left\{e_{0}, e_{2}, e_{3}\right\}$ and $\left(e_{j}, l_{0}^{\prime}\right)=0(j=0,1,2)$ $\left(e_{3}, \tilde{l}_{0}^{\prime}\right) \neq 0$. 
$\beta$. Suppose now $\operatorname{dim} I / C=2$. Using the notations introduced before, we denote by $V$ the subspace, spanned by $\gamma_{1}, \gamma_{2}$, and $\delta$, of $\mathscr{L}^{\prime}$. We are going to prove that, if $\operatorname{dim} V<3$, there exists a choice of $\left\{l_{1}, l_{2}\right\}$, such that $\gamma_{i} \equiv 0(i=1,2)$. In fact, since $\delta \not \equiv 0$, we have $\operatorname{dim} V>0$. If $\operatorname{dim} V=1$ we have $\gamma_{j}=d_{j} \delta(j=1,2)$, hence it suffices to replace $\left\{l_{1}, l_{2}\right\}$ by $\left\{l_{1}+x_{1} c, l_{2}+x_{2} c\right\}$, where $\left\{x_{1}, x_{2}\right\}$ satisfy $d_{1}=x_{1}-\alpha x_{2}$, and $d_{2}=-\alpha x_{1}+x_{2}$. If $\operatorname{dim} V=2$, the map $\psi(l)=-\left(\left.(\operatorname{ad} l)\right|_{l}\right)^{\prime}(l \in \mathscr{L})$ defines a homomorphism of $\mathscr{L}$ into the 3 dimensional algebra $\hat{\mathscr{L}}$ considered in the proof of Lemma 2 , such that $\operatorname{dim} \psi(\mathscr{L})=2$ and, because of $\delta \not \equiv, \psi(\mathscr{L}) \neq\left\{e_{1}, e_{2}\right\}$ which is impossible. Replacing $\mathscr{L}$ by $\hat{H}$, and using $H \nsubseteq \mathscr{L}_{0}$ we conclude that $H$ is properly contained in $H_{\delta} \supseteqq H_{\gamma}$, and $\operatorname{dim} H / H_{\gamma} \neq 1, H_{\delta} \neq H$ is impossible. If $\operatorname{dim} H / H_{\gamma}=2$, no nonzero element $a_{1} l_{1}+a_{2} l_{2}$ can belong to $H$. In fact, otherwise we would have

$$
0=\left(\left[h, a_{1} l_{1}+a_{2} l_{2}\right], l_{0}^{\prime}\right)=\left(a_{1} \gamma_{1}(h)+a_{2} \gamma_{2}(h)\right)\left(c, l_{0}^{\prime}\right)
$$

since $H<l_{0}^{\prime}$ and $\left(l_{j}, l_{0}^{\prime}\right)=0(j=1,2)$.

But this gives $a_{1}=a_{2}=0$, by virtue of $\left(c, l_{0}^{\prime}\right) \neq 0$, and the independence of $\left\{\gamma_{1}, \gamma_{2}\right\}$ on $H$. Summing up, we have to consider the following subcases.

a. $\operatorname{dim} H / H_{\gamma}=2$ and $H_{\delta}=H$. Observe, that in this case evidently $[H, H] \subset Z$, hence also $[H, H] \subset S$. We can write $H=X_{1}+X_{2}+C+S$ with $X_{j}=\left\{x_{j}\right\}$, $\gamma_{i}\left(x_{j}\right)=\delta_{i j}$ and $x_{i} \perp l_{0}^{\prime}(i, j=1,2)$. Therefore $\tilde{\mathscr{L}}=\left\{e_{1}, e_{2}, e_{3}, e_{4}, e_{5}\right\}$, where $\left[e_{1}, e_{2}\right]=e_{5}$, $\left[e_{3}, e_{4}\right]=e_{5}, \tilde{H}=\left\{e_{1}, e_{2}, e_{5}\right\}, \tilde{H}_{1}=\left\{e_{3}, e_{4}, e_{5}\right\}$ and $\left(e_{j}, \tilde{l}_{0}^{\prime}\right)=0(j=1,2,3,4)$.

b. $\operatorname{dim} H / H_{\gamma}=2$ and $H_{\gamma} \cap H_{\delta}$ is properly contained in $H_{\gamma}$. We have

$$
H=X_{1}+X_{2}+Y+C+S,
$$

where the $x_{i}$, beside conditions as in (a) satisfy $\delta\left(x_{j}\right)=0(j=1,2)$. Furthermore $Y=\{y\}, \delta(y)=1, \gamma_{j}(y)=0(j=1,2)$, and $\left(y, l_{0}^{\prime}\right)=0$. To obtain the brackets among $\left\{x_{1}, x_{2}, y\right\}$ we note, that the map $\psi$ considered above, when restricted to $H$, establishes an isomorphism between $H /(S+C)$ and $\hat{\mathscr{L}}$, and that, with the notations of the proof of Lemma $2, \psi(h)=\left(-\delta(h),-\gamma_{1}(h),-\gamma_{2}(h)\right)(h \in H)$. We have therefore $\left[y, x_{1}\right]=-x_{1}+\alpha x_{2},\left[y_{1} x_{2}\right]=-\alpha x_{1}-x_{2} \bmod (S+C)$ and hence, because of $H<l_{0}^{\prime}$, also mod $(S)$. We conclude in this fashion, that $\tilde{\mathscr{L}}=\left\{e_{1}, e_{2}, e_{3}, e_{4}, e_{5}\right\}$ with the nonvanishing brackets $\left[e_{0}, e_{1}\right]=-e_{1}+\alpha e_{2},\left[e_{0}, e_{2}\right]=-\alpha e_{1}-e_{2},\left[e_{0}, e_{3}\right]=-e_{3}+\alpha e_{4}$, $\left[e_{0}, e_{4}\right]=\alpha e_{3}+e_{4},\left[e_{1}, e_{3}\right]=e_{5},\left[e_{2}, e_{4}\right]=e_{5}$. Furthermore $\tilde{H}=\left\{e_{0}, e_{1}, e_{2}, e_{5}\right\}$, $\tilde{H}_{1}=\left\{e_{0}, e_{3}, e_{4}, e_{5}\right\}$ and $\left(e_{j}, I_{0}^{\prime}\right) \neq 0$ only for $j=5$.

c. $\operatorname{dim} H / H_{y}=1, H_{\delta}=H$. We have in this case $a_{1} \gamma_{1}+a_{2} \gamma_{2} \equiv 0$ on $H$, where $a_{1}^{2}+a_{2}^{2} \neq 0$. Replacing $\left\{l_{1}, l_{2}\right\}$ by $\left\{a_{1} l_{1}+a_{2} l_{2},-a_{2} l_{1}+a_{1} l_{2}\right\}$ we can suppose $\gamma_{1} \equiv 0$ on $H$, and we have then $\left[h, l_{1}\right]=0(h \in H)$. As shown above, $H$ contains its centralizer in $\mathscr{L}$ if either $H=(J)$, or ind $\left(H_{1} l_{0}^{\prime}\right)$ is irreducible, and therefore $l_{1} \in H$. On the other hand, $\gamma_{2} \neq \equiv$ implies $l_{2} \bar{\epsilon} H$. Proceeding, as in $\alpha \cdot a$, we conclude, that $\tilde{\mathscr{L}}=\left\{e_{1}, e_{2}, e_{3}\right\},\left[e_{1}, e_{2}\right]=e_{3}, \tilde{H}=\left\{e_{1}, e_{3}\right\}, \tilde{H}_{1}=\left\{e_{2}, e_{3}\right\}$ and $\left(e_{j}, l_{0}^{\prime}\right)=0(j=1,2)$.

The statement ind $\left(\tilde{H}, l_{0}^{\prime}\right)=$ ind $\left(\tilde{H}_{1}, \tilde{l}_{0}^{\prime}\right)$ is proved for cases $\alpha . \mathrm{a}, \beta$. a, and $\beta$. c in [1, Lemma 5, Chapitre II], and for cases $\alpha . \mathrm{b}$ and $\beta$. b in [1, Lemma 7, Chapitre II]. 
To prove, that all subalgebras listed above possess property $J$, by virtue of Proposition 1 it suffices to show, that they have property $G$. We shall confine ourselves to verify $\tilde{H}=(G)$ in case $\beta . \mathrm{b}$; the rest can be settled in a similar fashion. We write $\left(e_{5}, \tilde{l}_{0}^{\prime}\right)=x \neq 0$. Using a basis, dual to $\left\{e_{j}\right\}$, in $\tilde{\mathscr{L}}^{\prime}$, we get $\tilde{l}_{0}^{\prime}+\tilde{H}^{\perp}=\left\{\left(0,0,0, y, y_{2}, x\right)\right\}$, where $y_{1}$ and $y_{2}$ are arbitrary real numbers. If $\tilde{H}$ is not maximal self orthogonal with respect to an $l^{\prime}$ in $I_{0}^{\prime}+\widetilde{H}^{\perp}$, then there exists a nonzero element $e$ of the form $a_{3} e_{3}+a_{4} e_{4}$, such that $0=\left([h, e], \tilde{l}^{\prime}\right)$ for all $h$ in $\tilde{H}$. Hence, in particular

$$
0=\left(\left[e_{1}, e\right], l^{\prime}\right)=a_{3} x, \quad 0=\left(\left[e_{2}, e\right], l^{\prime}\right)=a_{4} x,
$$

contradicting $e \neq 0$.

2. To finish the proof of Proposition 2, we suppose first, that ind $\left(H, l_{0}^{\prime}\right)$ is irreducible, and prove, that this implies $H=(J)$. According to 1 above, it suffices to consider the case, when $H \subset \mathscr{L}_{0}$. But then ind $\left(H, l_{0}^{\prime} ; \mathscr{L}_{0}\right)$, too is irreducible and hence, by induction, $H=\left(J, \mathscr{L}_{0}\right)$. Therefore to obtain the desired conclusion, it is enough to check, that $\mathscr{L}_{0}$ satisfies the conditions of Lemma $1^{\prime}$ and Lemma $2^{\prime}$ resp. We recall, that with the notations used above $\operatorname{dim} \mathscr{L} / \mathscr{L}_{0}=\operatorname{dim} I / C=1$ or 2 .

$\alpha$. If $\operatorname{dim} I / C=1$, suppose, that $r$ is a nonzero element of $R\left(l_{0}^{\prime}+l_{0}^{1}\right)-\mathscr{L}_{0}$, where $l_{0}^{\perp}$ is arbitrary in $\mathscr{L}_{0}^{\perp}$. Then, in particular, since $l_{0} \in \mathscr{L}_{0}, 0=\left(\left[r, l_{0}\right], l_{0}^{\perp}\right)=\gamma(r)\left(c_{1} l_{0}^{\prime}\right)$, and so $\gamma(r)=0$ and $r \in \mathscr{L}_{0}$.

$\beta$. Assume now, that $\operatorname{dim} I / C=2$. Since $\left(l_{j}, l_{0}^{\prime}\right)=0(j=1,2)$, we have

$$
\left(\left[l, l_{j}\right], l_{0}^{\prime}\right)=\gamma_{j}(l)\left(c, l_{0}^{\prime}\right) \quad(j=1,2)
$$

for all $l$ in $\mathscr{L}$. Since $\left(c, l_{0}^{\prime}\right) \neq 0$, we conclude from this first, that $R \cong R_{0} \subset \mathscr{L}_{0}$, and secondly, by virtue of the independence of $\gamma_{1}$ and $\gamma_{2}$, that $\operatorname{dim} R+2=\operatorname{dim} R_{0}$.

3. Assume finally, that $H=(J)$. To prove that this implies that ind $\left(H, l_{0}^{\prime}\right)$ is irreducible by 1 it suffices again to consider the case when $H$ is contained in $\mathscr{L}_{0}$. Since we have also $H=\left(J, \mathscr{L}_{0}\right)$ we can conclude by induction, that ind $\left(H, l_{0}^{\prime} ; \mathscr{L}_{0}\right)$ is irreducible, and then finish the proof exactly as at the end of $\beta$ by observing (cf. the beginning of $c$ ) that the stabilizer of $\pi l_{0}^{\prime}$ in $\mathscr{L}$ is $\mathscr{L}_{0}$ (for the computation of the orbits cf. again [9, p. 156]).

The following Lemma follows from Proposition 2 above and from Théorème, Chapitre III in [1]; our purpose here is to give a direct proof, independent of representation theory.

Lemma 5. The subalgebra $H$ is of a maximal dimension in the family of all subalgebras subordinated to $l_{0}^{\prime}$ if and only if $\operatorname{dim} H=\operatorname{dim} \mathscr{L}-\frac{1}{2} \operatorname{dim} O$, where $O$ is the orbit of $l_{0}^{\prime}$.

Proof. If $H<l_{0}^{\prime}, H$ is self orthogonal with respect to $l_{0}^{\prime}$, and therefore $\operatorname{dim} H \leqq \frac{1}{2}(\operatorname{dim} \mathscr{L}+\operatorname{dim} O)=\operatorname{dim} \mathscr{L}-\frac{1}{2} \operatorname{dim} O$. In this fashion it suffices to show, that the value on the right hand side is assumed by an $H, H<l_{0}^{\prime}$. In what follows ve prove the existence of an $H$ with $H=(J)$, which implies the desired conclusion. We proceed by induction and assume the validity of the statement to be proved for 
algebras of dimension less than $\operatorname{dim} \mathscr{L}$. Using the notations of the proof of Proposition 2 , if we have case A, then $\tilde{\mathscr{L}}=\mathscr{L} \mid I$, by induction, contains a subalgebra $\tilde{H}$ of the desired sort. By Lemma 4 , its complete inverse image $H$ in $\mathscr{L}$ satisfies $H=(J)$. For each of the subcases of cases $B$ and $C$ we have constructed a subalgebra $\mathscr{L}_{0}$, of codimension 1 or 2 , of $\mathscr{L}$, satisfying the conditions of Lemma $1^{\prime}$ or Lemma $2^{\prime}$ resp. Therefore to finish the proof of our Lemma it suffices to choose, using the hypothesis of our induction, a subalgebra having the property $J$, of $\mathscr{L}_{0}$.

REMARK. Observe, that the above proof yields also the following result: Any orbit $O$ of the representation, contragredient to the adjoint representation, contains a linear variety of $\operatorname{dimension} \frac{1}{2} \operatorname{dim} O$. In fact, it suffices to note, that if $H=(J)$ then, by definition, $l_{0}^{\prime}+H^{\perp} \subset O$, and that

$$
\operatorname{dim} H^{\perp}=\operatorname{dim} \mathscr{L}-\operatorname{dim} H=\frac{1}{2}(\operatorname{dim} \mathscr{L}-\operatorname{dim} R)=\frac{1}{2} \operatorname{dim} O .
$$

Combining Proposition 2 with Lemma 5, we obtain the following:

THEOREM 1. Suppose, that $G$ is an exponential group with the Lie algebra $\mathscr{L}$, and let $l_{0}^{\prime}$ be a nonzero element of the dual $\mathscr{L}^{\prime}$ of $\mathscr{L}$. Denote by $O$ the orbit, containing $l_{0}^{\prime}$, of the representation, contragredient to the adjoint representation of $G$. Suppose, that $H$ is a subalgebra of $\mathscr{L}$, such that its first derived algebra is orthogonal to $l_{0}^{\prime}$ with respect to the canonical bilinear form on $\mathscr{L} \times \mathscr{L}^{\prime}$. Then the character

$$
\chi(\exp h)=\exp \left[i\left(h, l_{0}^{\prime}\right)\right] \quad(h \in H)
$$

induces an irreducible representation of $G$ if and only if the dimension of $H$ is maximal in the family of subalgebras satisfying the condition above, and if the linear variety $l_{0}^{\prime}+\left(\right.$ orthogonal complement of $H$ in $\left.\mathscr{L}^{\prime}\right)$ is contained in $\mathscr{L}^{\prime}$.

5. The purpose of this section is to give two sufficient conditions, under which a subalgebra $H$, subordinated to $l_{0}^{\prime}$ and satisfying $\operatorname{dim} H=\operatorname{dim} \mathscr{L}-\frac{1}{2} \operatorname{dim} O$ (cf. Lemma 5) has the property $J$ too.

We recall (cf. Introduction), that an exponential algebra $\mathscr{L}$ is called quasinilpotent, if it has no nonzero real roots. In particular, every nilpotent algebra is quasi-nilpotent.

Proposition 3. Suppose, that $\mathscr{L}$ is quasi-nilpotent, and that the subalgebra $H$, subordinated to $l_{0}^{\prime} \in \mathscr{L}^{\prime}$, satisfies $\operatorname{dim} H=\operatorname{dim} \mathscr{L}-\frac{1}{2} \operatorname{dim} O$. Then we have also $H=(J)$.

Proof. The statement being clear if $\operatorname{dim} \mathscr{L}=1$, in what follows we shall assume $\operatorname{dim} \mathscr{L}>1$, and suppose the validity of the proposition for algebras of smaller dimension. Also, we can assume $\operatorname{dim} H<\operatorname{dim} \mathscr{L}$ since otherwise $H$ clearly has property $J$. We denote by $\mathscr{L}_{0}$ a subalgebra, containing $H$, of $\mathscr{L}$, such that either $\operatorname{dim} \mathscr{L}_{0}+1=\operatorname{dim} \mathscr{L}$ or $\operatorname{dim} \mathscr{L}_{0}+2=\operatorname{dim} \mathscr{L}$ and $\mathscr{L} \mid \mathscr{L}_{0}=$ irr $\left(\mathscr{L}_{0}\right)$ (cf. Remark after Lemma 2). If $\operatorname{dim} H=\operatorname{dim} \mathscr{L}-\frac{1}{2} \operatorname{dim} O$ then $H$ is maximal self orthogonal with respect to $B\left(l_{1}, l_{2} ; l_{0}^{\prime}\right)$, hence also with respect to $B\left(l_{1}, l_{2} ; \mathscr{L}_{0}, l_{0}^{\prime}\right)$ (cf. $\left.2 \mathrm{~b}\right)$. From this 
we conclude, that $R \subseteq R_{0} \subseteq \mathscr{L}_{0}$, and that, by induction, $H=\left(J, \mathscr{L}_{0}\right)$. Therefore it suffices to verify, that in each case $\mathscr{L}_{0}$ satisfies the conditions of Lemma $1^{\prime}$ and Lemma $2^{\prime}$ resp. If the codimension of $\mathscr{L}_{0}$ in $\mathscr{L}$ is 1 , then it is enough to observe, that it is necessarily an ideal of $\mathscr{L}$. In fact, if $l_{1}$ is a nonzero element not belonging to $\mathscr{L}_{0}$, and if $l_{0}$ is arbitrary in $\mathscr{L}_{0}$, we have ad $l_{0}\left(l_{1}\right) \equiv \alpha l_{1}\left(\bmod \mathscr{L}_{0}\right)$. Since $\alpha$ is a real eigenvalue of the operator ad $l_{0}$, and since $\mathscr{L}$ is quasi-nilpotent, we have $\alpha=0$, proving $\left[\mathscr{L}, \mathscr{L}_{0}\right] \subseteq \mathscr{L}_{0}$. If $\operatorname{dim} \mathscr{L}_{0}+2=\operatorname{dim} \mathscr{L}$ then, as observed above, $R \cong R_{0} \subseteq H$ and $\operatorname{dim} H=\frac{1}{2}\left(\operatorname{dim} \mathscr{L}_{0}+\operatorname{dim} R_{0}\right)=\frac{1}{2}(\operatorname{dim} \mathscr{L}+\operatorname{dim} R)$ and thus $\operatorname{dim} R+2=\operatorname{dim} R_{0}$.

The following statement is an immediate consequence of Proposition 2 and Proposition 3, along with Lemma 5.

Corollary (Bernat). Suppose, that $\mathscr{L}$ is quasi-nilpotent, and that the dimension of the subalgebra $H$ is maximal among the subalgebras subordinated to $l_{0}^{\prime}$. Then ind $\left(H, l_{0}^{\prime}\right)$ is irreducible.

Proposition 4. Suppose, that the dimension of the subalgebra $H$ of the exponential algebra $\mathscr{L}$ is maximal among the subalgebras subordinated to the nonzero element $l_{0}^{\prime}$ of $\mathscr{L}^{\prime}$. Suppose also, that the orbit $O$ of $l_{0}^{\prime}$ is closed. Then ind $\left(H, l_{0}^{\prime}\right)$ is irreducible.

Proof. By virtue of Proposition 2, it suffices to show, that the previous assumptions imply, that $H=(J)$. Since by Lemma 5 we have $\operatorname{dim} H=\operatorname{dim} \mathscr{L}-\frac{1}{2} \operatorname{dim} O$, it is enough to prove, that $l_{0}^{\prime}+H^{\perp} \subset O$. By the assumption on $O$, the intersection of $O$ and of $l_{0}^{\prime}+H^{\perp}$ is closed, hence it suffices to show, that at the same time it is open in $l_{0}^{\prime}+H^{\perp}$. Let $h_{0}^{\perp}$ be an element of $H^{\perp}$, such that $l_{0}^{\prime}+h_{0}^{\perp}$ belongs to $O$, and consider the map $\phi$ from $H$ into $O \cap\left(l_{0}^{\prime}+H^{\perp}\right)$ defined by $\phi(\bar{h})=\delta(\bar{h})\left(l_{0}^{\prime}+h_{0}^{\perp}\right)(h \in H)$. Evidently it is enough to prove, that the rank $r$ of $\phi$ at $h=0$ is not smaller, than $\operatorname{dim} H^{\perp}=\operatorname{dim} \mathscr{L}-\operatorname{dim} R$. But using the reasoning of $A$ of the proof of Proposition 1 we conclude, that

$$
\begin{aligned}
r & =\operatorname{dim} H-\operatorname{dim}\left(H \cap R\left(l_{0}^{\prime}+h_{0}^{\perp}\right)\right) \geqq \operatorname{dim} H-\operatorname{dim} R\left(l_{0}^{\prime}+h_{0}^{\perp}\right) \\
& =\operatorname{dim} H-\operatorname{dim} R\left(l_{0}^{\prime}\right)=\operatorname{dim} H^{\perp},
\end{aligned}
$$

since $l_{0}^{\prime}+h_{0}^{\perp} \in O$ implies $\operatorname{dim} R=\operatorname{dim} R\left(l_{0}^{\prime}+h_{0}^{\perp}\right)$.

6. In this section we suppose, that $\mathscr{L}$ has real roots. The motivation for the following considerations is described at the end of the Introduction.

Let $H$ be a subalgebra, subordinated to $l_{0}^{\prime} \in \mathscr{L}^{\prime}$, of $\mathscr{L}$, and $\bar{H}$ the connected subgroup corresponding to $H$. We define again a character $\chi$ on $\bar{H}$ by $\chi(\exp h)$ $=\exp \left[i\left(h, l_{0}^{\prime}\right)\right]$. Let $x$ be a fixed element of $\overline{\mathscr{L}}$. We have $\chi\left(x^{-1} \exp h x\right)=\chi(\exp h)$ for all $\exp h \in x \bar{H} x^{-1} \cap \bar{H}$ if and only if $\left(\sigma\left(x^{-1}\right) h, l_{0}^{\prime}\right) \equiv\left(h, l_{0}^{\prime}\right)$ for all $h \in \sigma(x)(H) \cap H$; but this is equivalent to $\delta(x) l_{0}^{\prime}-l_{0}^{\prime} \in(\sigma(x)(H) \cap H)^{\perp}=\delta(x)\left(H^{\perp}\right)+H^{\perp}$. This leads to the following

Definition 4. We say, that the subalgebra $H$, subordinated to $l_{0}^{\prime}$, has the property $C$, and write $H=(C)$ if $\delta(x)\left(l_{0}^{\prime}+h_{1}^{\perp}\right)=l_{0}^{\prime}+h_{2}^{\perp}\left(h_{1}^{\perp}, h_{2}^{\perp} \in H^{\perp}\right)$ implies $x \in \bar{H}$.

Proposition 5. We have $H=(C)$ if and only if $H=(J)$. 
Proof. A. $(J) \Rightarrow(C)$. By virtue of Proposition 1 it suffices to show, that $H=(F)$ implies $H=(C)$. If $h_{j}^{\perp}(j=1,2)$ are elements in $H^{\perp}$, we can find elements $h_{k}$ in $H$, such that $\delta\left(\bar{h}_{k}\right) l_{0}^{\prime}=l_{0}^{\prime}+h_{k}^{\perp}(k=1,2)$. Hence $\delta(x)\left(l_{0}^{\prime}+h_{1}^{\perp}\right)=l_{0}^{\prime}+h_{2}^{\perp}$ implies

$$
\delta\left(\bar{h}_{2}^{-1} x \bar{h}_{1}\right) l_{0}^{\prime}=l_{0}^{\prime}
$$

and therefore $\bar{h}_{2}^{-1} x \bar{h}_{1}$ belongs to the connected subgroup corresponding to the radical $R$ of $l_{0}^{\prime}$. But if $H=(F)$ then, in particular, $H$ is maximal self orthogonal with respect to $l_{0}^{\prime}$, and thus $R \subset H$. Hence finally $\bar{h}_{2}^{-1} x \bar{h}_{1} \in \bar{R} \subset \bar{H}$ and $x \in \bar{H}$.

B. $(C) \Rightarrow(J)$. Again by Proposition 1 it suffices to prove, that $H=(C)$ implies $H=(G)$. We are going to do this by showing, that if $H=(C)$, then $H$ is maximal self orthogonal with respect to $l_{0}^{\prime}$. In fact, it will suffice then to observe, that $H<l_{0}^{\prime}$ implies $H<l_{0}^{\prime}+h^{\perp}$ for any $h^{\perp}$ in $H^{\perp}$, and thus the condition $H=(C)$ remains the same upon replacing $l_{0}^{\prime}$ by $l_{0}^{\prime}+h_{0}^{\perp}\left(h_{0}^{\perp} \in H^{\perp}\right)$.

Since the roots of $\mathscr{L}$ are real, there exists a subalgebra $\mathscr{L}_{0}$, of codimension 1 , of $\mathscr{L}$, containing $H\left({ }^{2}\right)$. Next we prove, that $H$ has the property $C$ with respect to $\mathscr{L}_{0}$, or that $H=\left(C, \mathscr{L}_{0}\right)$. We denote by $\pi$ the projection of $\mathscr{L}^{\prime}$ onto $\mathscr{L}_{0}^{\prime}=\mathscr{L}^{\prime} \mid \mathscr{L}_{0}^{\perp}$, and observe, that the orthogonal complement of $H$ in $\mathscr{L}_{0}^{\prime}$ is the image of $H^{\perp}$ under $\pi$. Furthermore, if $\delta_{0}$ is the representation, corresponding to $\delta$, of $\overline{\mathscr{L}}_{0}$ on $\mathscr{L}_{0}^{\prime}$, we have $\delta_{0}\left(\bar{l}_{0}\right) \pi=\pi \delta\left(\bar{l}_{0}\right)$ for all $l_{0}$ in $\mathscr{L}_{0}$. Therefore, if $H \neq\left(C, \mathscr{L}_{0}\right)$, there exist elements $h_{j}^{\perp}(j=1,2)$ in $H^{\perp}$, and $l_{0} \in \mathscr{L}_{0}$ not in $H$ such that

$$
\delta_{0}\left(\bar{l}_{0}\right) \pi\left(l_{0}^{\prime}+h_{1}^{\perp}\right)=\pi\left(l_{0}^{\prime}+h_{2}^{\perp}\right) .
$$

By the previous remarks, this implies

$$
\delta\left(\bar{l}_{0}\right)\left(l_{0}^{\prime}+h_{1}^{\perp}\right)=l_{0}^{\prime}+h_{2}^{\perp}+l_{0}^{\perp},
$$

where $l_{0}^{\perp}$ is orthogonal to $\mathscr{L}_{0}$. Since $h_{2}^{\perp}+l_{0}^{\perp} \in H^{\perp}$, and $l_{0} \bar{\epsilon} H$, we have then a contradiction to $H=(C)$. Proceeding by induction, we conclude, that $H$ is maximal self orthogonal in $\mathscr{L}_{0}$, or $\operatorname{dim} H=\frac{1}{2}\left(\operatorname{dim} \mathscr{L}_{0}+\operatorname{dim} R_{0}\right)$. Since $H=(C)$ trivially implies, that $R \subset H, R$ is of codimension 1 in $R_{0}$, and $\operatorname{dim} H=\frac{1}{2}(\operatorname{dim} \mathscr{L}+\operatorname{dim} R)$, proving, that $H$ is maximal self orthogonal in $\mathscr{L}$.

Combining Proposition 5 with Proposition 2 we obtain the following.

THEOREM 2. Let $G$ be an exponential group with real roots, $\Gamma$ a closed connected subgroup of $G$, and $\chi$ a continuous homomorphism of $\Gamma$ into the multiplicative group of complex numbers of absolute value one. For an element $x$ of $G$, we denote by $\Gamma_{x}$ the intersection of the subgroups $\Gamma$ and $x^{-1} \Gamma x$, and by $\chi_{x}$ the character of $\Gamma_{x}$ defined by $\chi_{x}(\gamma)=\chi\left(x y x^{-1}\right)\left(\gamma \in \Gamma_{x}\right)$. Then the representation, induced by $\chi$, of $G$ is irreducible if and only if, for all $x$ not belonging to $\Gamma$, the character $\chi_{x}$ is different from the restriction of $\chi$ to $\Gamma_{x}$.

${ }^{2}$ ) This is the only point, where the hypothesis of the real roots is used. Although likely to be valid, the author could not prove Theorem 2 without this restriction. 
Proof. We denote by $\mathscr{L}$ the Lie algebra of $G$. Then $\Gamma$ is the image, through the exponential map, of some subalgebra $H$ of $\mathscr{L}$. According to what precedes, to obtain the desired result it suffices to observe, that $\chi$ can be written as $\exp \left[i\left(h, l_{0}^{\prime}\right)\right]$ $(h \in H)$ with an appropriately chosen $l_{0}^{\prime}$ in $\mathscr{L}^{\prime}$.

REMARK. As observed earlier (cf. the end of the Introduction), Theorem 2 extends to certain exponential groups a situation known for finite groups (cf. [5, Theorem 6]).

\section{BIBLIOGRAPHY}

1. P. Bernat, Sur le dual d'un groupe exponentiel résoluble, Thèse, Paris, 1965.

2. J. Dixmier, L'application exponentielle dans les groupes de Lie résolubles, Bull. Soc. Math. France 85 (1957), 113-121.

3. A. A. Kirillov, Unitary representations of nilpotent Lie groups, Uspehi Mat. Nauk 17 no. 4, (1962), 57-110= Russian Math. Surveys 17 (1962), 53-104.

4. G. W. Mackey, Imprimitivity for representations of locally compact groups, Proc. Nat. Acad. Sci. U.S.A. 35 (1949), 537-545.

5. — Induced representations of groups, Amer. J. Math. 73 (1951), 576-592.

6. - Induced representations of locally compact groups, Ann. of Math. 55 (1952), 101-139.

7. L. Pukánszky, Leçons sur la représentation des groupes, Dunod, Paris, 1966.

8. M. Saito, Sur certains groupes de Lie résolubles. I, II, Sci. Papers College Gen. Ed. Univ. Tokyo 7 (1957), 1-11; 157-168.

9. O. Takenouchi, Sur la facteur représentation des groupes de Lie de type (E), Math. J. Okayama Univ. 7 (1957), 151-161.

University of Pennsylvania, Philadelphia, Pennsylvania 